\title{
Novel induction of CD40 expression by tumor cells with RAS/RAF/PI3K pathway inhibition augments response to checkpoint blockade
}

Chi Yan ${ }^{1,2}$, Nabil Saleh ${ }^{1,2}$, Jinming Yang ${ }^{1,2}$, Caroline A. Nebhan ${ }^{1,2,3}$, Anna E. Vilgelm ${ }^{4}$, E. Premkumar Reddy ${ }^{5}$ Joseph T. Roland ${ }^{6}$, Douglas B. Johnson ${ }^{3}$, Sheau-Chiann Chen ${ }^{7}$, Rebecca L. Shattuck-Brandtt,

Gregory D. Ayers ${ }^{7}$ and Ann Richmond ${ }^{1,2^{*}}$

\begin{abstract}
Background: While immune checkpoint blockade (ICB) is the current first-line treatment for metastatic melanoma, it is effective for $\sim 52 \%$ of patients and has dangerous side effects. The objective here was to identify the feasibility and mechanism of RAS/RAF/PI3K pathway inhibition in melanoma to sensitize tumors to ICB therapy.

Methods: Rigosertib (RGS) is a non-ATP-competitive small molecule RAS mimetic. RGS monotherapy or in combination therapy with ICB were investigated using immunocompetent mouse models of BRAF ${ }^{\mathrm{wt}}$ and BRAF ${ }^{\text {mut }}$ melanoma and analyzed in reference to patient data.

Results: RGS treatment (300 mg/kg) was well tolerated in mice and resulted in $~ 50 \%$ inhibition of tumor growth as monotherapy and $\sim 70 \%$ inhibition in combination with aPD1 + aCTLA4. RGS-induced tumor growth inhibition depends on CD40 upregulation in melanoma cells followed by immunogenic cell death, leading to enriched dendritic cells and activated T cells in the tumor microenvironment. The RGS-initiated tumor suppression was partially reversed by either knockdown of CD40 expression in melanoma cells or depletion of CD8 ${ }^{+}$cytotoxic $T$ cells. Treatment with either dabrafenib and trametinib or with RGS, increased $\mathrm{CD} 40^{+} \mathrm{SO} \times 10^{+}$melanoma cells in the tumors of melanoma patients and patient-derived xenografts. High CD40 expression level correlates with beneficial T-cell responses and better survival in a TCGA dataset from melanoma patients. Expression of CD40 by melanoma cells is associated with therapeutic response to RAF/MEK inhibition and ICB.

(Continued on next page)
\end{abstract}

\footnotetext{
* Correspondence: ann.richmond@vanderbilt.edu

CONTACT FOR REAGENT AND RESOURCE SHARING

Further information and requests for resources and reagents should be

directed to and will be fulfilled according to institutional rules by the Lead

Contact, Ann Richmond (ann.richmond@vanderbilt.edu).

${ }^{1}$ Department of Veterans Affairs, Tennessee Valley Healthcare System, 432

PRB, 2220 Pierce Ave, Nashville, TN 37232, USA

${ }^{2}$ Department of Pharmacology, Vanderbilt University School of Medicine,

Nashville, TN, USA

Full list of author information is available at the end of the article
}

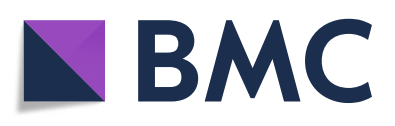

(- The Author(s). 2021 Open Access This article is licensed under a Creative Commons Attribution 4.0 International License, which permits use, sharing, adaptation, distribution and reproduction in any medium or format, as long as you give appropriate credit to the original author(s) and the source, provide a link to the Creative Commons licence, and indicate if changes were made. The images or other third party material in this article are included in the article's Creative Commons licence, unless indicated otherwise in a credit line to the material. If material is not included in the article's Creative Commons licence and your intended use is not permitted by statutory regulation or exceeds the permitted use, you will need to obtain permission directly from the copyright holder. To view a copy of this licence, visit http://creativecommons.org/licenses/by/4.0/. The Creative Commons Public Domain Dedication waiver (http://creativecommons.org/publicdomain/zero/1.0/) applies to the data made available in this article, unless otherwise stated in a credit line to the data. 
(Continued from previous page)

Conclusions: Our data support the therapeutic use of RGS + aPD1 + aCTLA4 in RAS/RAF/PI3K pathway-activated melanomas and point to the need for clinical trials of RGS + ICB for melanoma patients who do not respond to ICB alone.

Trial registration: NCT01205815 (Sept 17, 2010).

Keywords: RAS/RAF/PI3K, CD40, Immunogenic cell death, Immune checkpoint blockade, Melanoma

\section{One-sentence summary}

RAS/RAF/PI3K inhibition promotes CD40 expression by melanoma cells, induces immunogenic cell death, and enhances response to immune checkpoint inhibitors.

\section{Introduction}

Approximately $20 \%$ of melanoma patient tumors harbor mutations in the neuroblastoma RAS viral oncogene homolog (NRAS), 60\% have a mutually exclusive mutation to NRAS in the v-raf murine sarcoma viral oncogene homolog B1 (BRAF), and 31\% have mutation in the phosphoinositide 3-kinase (PI3K) pathway $[1,2]$. These pathways are crucial to support melanoma cell proliferation, survival, or evasion of cell death. While BRAF ${ }^{\mathrm{V} 600}$ mut patients with unresectable or metastatic melanoma respond well to inhibitors of BRAF combined with MEK inhibitors [3, 4], a majority of patients will develop acquired resistance to these drugs through myriad of resistance mechanisms [5-7]. Recent advances in immune checkpoint blockade (ICB) therapy, such as targeting programmed cell death protein 1 (PD-1)/PD-ligand 1 (PD-L1) and/or cytotoxic T-lymphocyte-associated protein 4 (CTLA-4), illustrate the power of enhancing the patient's endogenous anti-melanoma immune responses $[4,8-10]$. Anti-PD-1 combined with $\alpha$-CTLA4 immunotherapy significantly prolong survival for $>52 \%$ of melanoma patients with previously untreated advanced melanoma (> 60 months median overall survival [OS]). Moreover, $44 \%$ of melanoma patients treated with $\alpha$-PD1 alone (19.9 months median OS) exhibit extended survival (36.9 months median OS) [10]. Currently, ICB is a first-line therapy for unresectable metastatic melanoma. However, many patients do not respond and adverse events can be severe and often deadly [11]. Therefore, improved targeted therapeutic approaches are needed, especially after patients have progressed on immunotherapy or targeted therapy alone.

For melanoma patients whose tumors harbor mutation in NRAS, treatment with BRAF inhibitors is contraindicated. However, MEK inhibitors alone or in combination with CDK4/6 inhibitors, or PI3K-AKT pathway inhibitors, resulted in partial responses in phase I/II clinical trials [12-14]. Rigosertib (RGS), also known as ON01910.Na, a non-ATP-competitive small molecule RAS mimetic, has the potential to block RAS-RAF-
MEK-ERK and PI3K-AKT-mTOR signaling pathways and to interfere with CRAF interaction with polo-like kinase 1 (PLK1) [15-17]. While multiple mechanisms of action of RGS have been reported, all studies have found it to be a potent inhibitor of tumor growth. RGS inhibits tumor growth in xenografts of colorectal and lung cancer and blocks tumor growth of a transgenic model of pancreatic cancers induced by K-RAS ${ }^{\mathrm{G} 12 \mathrm{D}}$ expression [18]. In head and neck squamous cell carcinoma, RGS inhibits the PI3K/mTOR pathway, blocks cell cycle and induces cytotoxicity through oxidative stress-induced reactive oxygen species (ROS) and activation of ERK/JNK pathways, resulting in increased phosphorylation and cytoplasmic translocation of ATF-2 [19]. In diffuse large B-cell lymphoma, RGS is reported to sequester the sumoylated c-MYB/TRAF2 complex in the cytoplasm, resulting in G1-cell cycle arrest and apoptosis [20]. Several phase I and phase II/III clinical trials have now been performed with RGS. These trials report modest toxicity, including trials in patients with myelodysplastic syndrome (MDS) [21], high-risk MDS after DNA methyltransferase inhibitor therapy [22], ovarian cancer [23], metastatic pancreatic cancer (with or without gemcitabine) [24], and advanced cancers [25]. Melanoma has not yet been a focus for clinical trials with RGS. Given the potential for NRAS/BRAF/PI3K mutant melanoma to be particularly sensitive to RGS, it is important to evaluate whether RGS is effective in preclinical models for the treatment of melanoma. Here we have linked response to RGS to expression of CD40 by melanoma tumor cells.

CD40, a cell surface molecule of the tumor necrosis factor (TNF) receptor family, was first identified on antigen-presenting cells (e.g., B cells, macrophages and dendritic cells $[\mathrm{DC}])$, and later shown to be expressed on the other cell types, including hematopoietic progenitors, platelets, eosinophils, T cells, epithelial cells, endothelial cells, fibroblasts and tumor cells [26]. In melanoma, CD40 is generally expressed by $30-50 \% \mathrm{hu}-$ man melanocytic lesions and melanoma cell lines [27]. Proinflammatory cytokines, such as interferon-gamma (IFNY) and tumor necrosis factor-alpha (TNF $\alpha$ ), and epigenetic modulators, such as histone deacetylase (HDAC) inhibitors, can induce CD40 expression on melanoma cells $[27,28]$. Importantly, the induction of CD40 on 
cancer cells, but not normal cells, promotes apoptotic and/or necrotic signaling, which results in the cell death of renal cell carcinoma [29], urothelial cell carcinoma [30], ovarian carcinoma [31, 32], cervical carcinoma [33] and bladder carcinoma [34]. Besides the direct cytotoxic effects in cancer cells, the ligation of CD40 on human melanoma cells also modulates tumor immunogenicity through upregulation of the expression of major histocompatibility complex (MHC) molecules and the production of proinflammatory factors (e.g., IL-6, IL-8 and TNFo, etc.) [35]. Furthermore, CD40 was shown to costimulate both anti-CD3-triggered $\mathrm{CD}^{+}{ }^{+}$and $\mathrm{CD} 8^{+} \mathrm{hu}$ man $\mathrm{T}$ cells [35] and enhance melanoma cell susceptibility to $\mathrm{T}$ cell lysis [28]. However, the regulation and function of CD40 in melanoma cells and its prognostic value in treatment responses remain to be examined.

Here, we report that RGS suppresses PI3K-mediated $\mathrm{AKT}^{\mathrm{T} 308}$ and mTORC2-mediated $\mathrm{AKT}^{\mathrm{Ser} 473}$ phosphorylation, increases CD40 expression and induces caspase3dependent melanoma cell death. RGS is well tolerated and effective in immunocompetent mouse melanoma models YUMM3.3 (BRAF ${ }^{\mathrm{mut}}$ ) and B16F10 (BRAF ${ }^{\mathrm{WT}}$ ). RGS significantly increases the frequency of plasmacytoid DC (pDC) and conventional DC (cDC) subsets in the tumor microenvironment (TME) and tumordraining lymph node (TDLN), respectively. In a dosedependent manner, RGS favors the proliferation and activation of tumor-infiltrating $\mathrm{T}$ cells (especially $\mathrm{CD} 8^{+}$ cytotoxic $\mathrm{T}$ cells $[\mathrm{Tc}])$, and the induction of NK cells, along with a reduction in M2-like macrophages in the TME. The anti-tumor effect of RGS is substantially abrogated in athymic mice bearing either the murine melanoma tumors or patient-derived xenografts (PDX). Remarkably, RGS exhibits synergistic effects with the combination of $\alpha$ PD-1/ $\alpha$ CTLA-4 ICB in B16F10 melanoma. At the mechanistic level, RGS monotherapy and in combination with ICB sensitizes tumors to immune activation via induced immunogenic cell death (ICD), associated with upregulation of costimulatory signals (such as CD40, CD80, and ICOS-L) on melanoma cells. We demonstrate here that RGS and BRAF/MEK inhibitors induce CD40 expression on murine melanoma cell lines and responsive patient melanoma cells in PDX models. Notably, we observe a significant induction of $\mathrm{CD} 40^{+} \mathrm{SOX} 0^{+}$melanoma cells in the tumors of melanoma patients post BRAF inhibitor treatment by multiplex IHC analysis. An extensive search of human cancer databases indicates that the gain of CD40/CD80/ICOSL copy number in melanoma patients is associated with significantly better survival. We also find that the levels of CD40 in human melanoma cells correlate to the response to RAF inhibitor and ICB treatments. Together, our work reveals a novel role of RAS/RAF/PI3K inhibition in ICD via promoting $\mathrm{CD} 40$ expression on melanoma cells as well as in inducing an anti-tumor immune response. Importantly this work provides preclinical evidence for the combination of RGS and ICB treatment as systemic therapy in patients with unresectable or metastatic melanoma.

\section{Results \\ Rigosertib induces melanoma cell death and inhibits melanoma tumor growth in vitro and in vivo}

We first evaluated the cytotoxic activity of RGS in four human and six murine melanoma cell lines with diverse genetic backgrounds, including cells with $N R A S^{\mathrm{Q} 61 R}$, BRAF $F^{\text {mut }}, P T E N^{\text {null }}, T P 53^{\text {null/mut }}$ and/or CDKN2A $A^{\text {null }}$ (Suppl.Table 1). Based on the CellTiter-Blue assay, the viability of all melanoma cell lines tested was significantly inhibited by micromolar concentrations of RGS (Fig. 1 a). Notably, while melanoma cells bearing either NRAS $^{\text {Q61R }}$ (SKMel2) or BRAF ${ }^{\text {mut }}$ (HS294T, SkMel5, A375, YUMM 5.2, YUMM 2.1 and YUMM 3.3) were sensitive to low dose RGS treatment at $0.1 \mu \mathrm{M}$, the NRAS $^{\text {WT }}{ }^{\text {BRAF }}{ }^{\mathrm{WT}}$ B16F10 cells were not sensitive to $0.1 \mu \mathrm{M}$ RGS, but were sensitive to $1 \mu \mathrm{M}$. By contrast, normal cells, including HEK293 cells and melanocytes from both human (Mel-ST) and murine (Melan-a with $\mathrm{BRAF}^{\mathrm{WT}}$ or $\mathrm{BRAF}^{\mathrm{mut}}$ ) origins, were totally refractory to RGS treatment. The dose-dependent cytotoxic effects of RGS on melanoma cells were further confirmed using crystal violet assay (Suppl.Fig. 1A). While BRAF ${ }^{\mathrm{V} 600 \mathrm{E}}$ is active independent of RAS binding, other signaling pathways that co-mediate melanoma growth remain dependent on RAS binding to the RAS-binding domain for activation. Given that BRAF ${ }^{\text {mut }}$ is the most common mutation in melanoma [36], we first focused on BRAFmut melanoma extensively for the following experiments. Using flow cytometric analyses, we observed that RGS triggered a dose- and time-dependent induction of apoptosis and necrosis in BRAF $^{\text {mut }}$ YUMM3.3 melanoma cells (Fig. 1 b).

Several studies have indicated that the ICD induction requires rapid generation of ROS and further ROS-based endoplasmic reticulum (ER) stress that contributes to antigen release and the induction of antitumor immunity [37]. We found that RGS promoted ROS production in both human SkMel5 and murine YUMM3.3 melanoma cells, and this effect reached a peak at $4 \mathrm{~h}$ posttreatment (Suppl.Fig. 1B). Interestingly, a prolonged ROS production $(\sim 24 \mathrm{~h}$ post-treatment) was observed in the highly sensitive YUMM3.3 cells (IC50 $=1.782 \mu \mathrm{M})$ as compared to the less sensitive SkMel5 cells (IC50= $60.05 \mu \mathrm{M})$. It was previously reported that RGS-induced ROS accumulation mediates ERK/JNK cascade activation in HeLa cells and head and neck cancer cell lines [19, 38]. However, ERK/JNK activation levels were not induced in the melanoma cells in response to RGS 


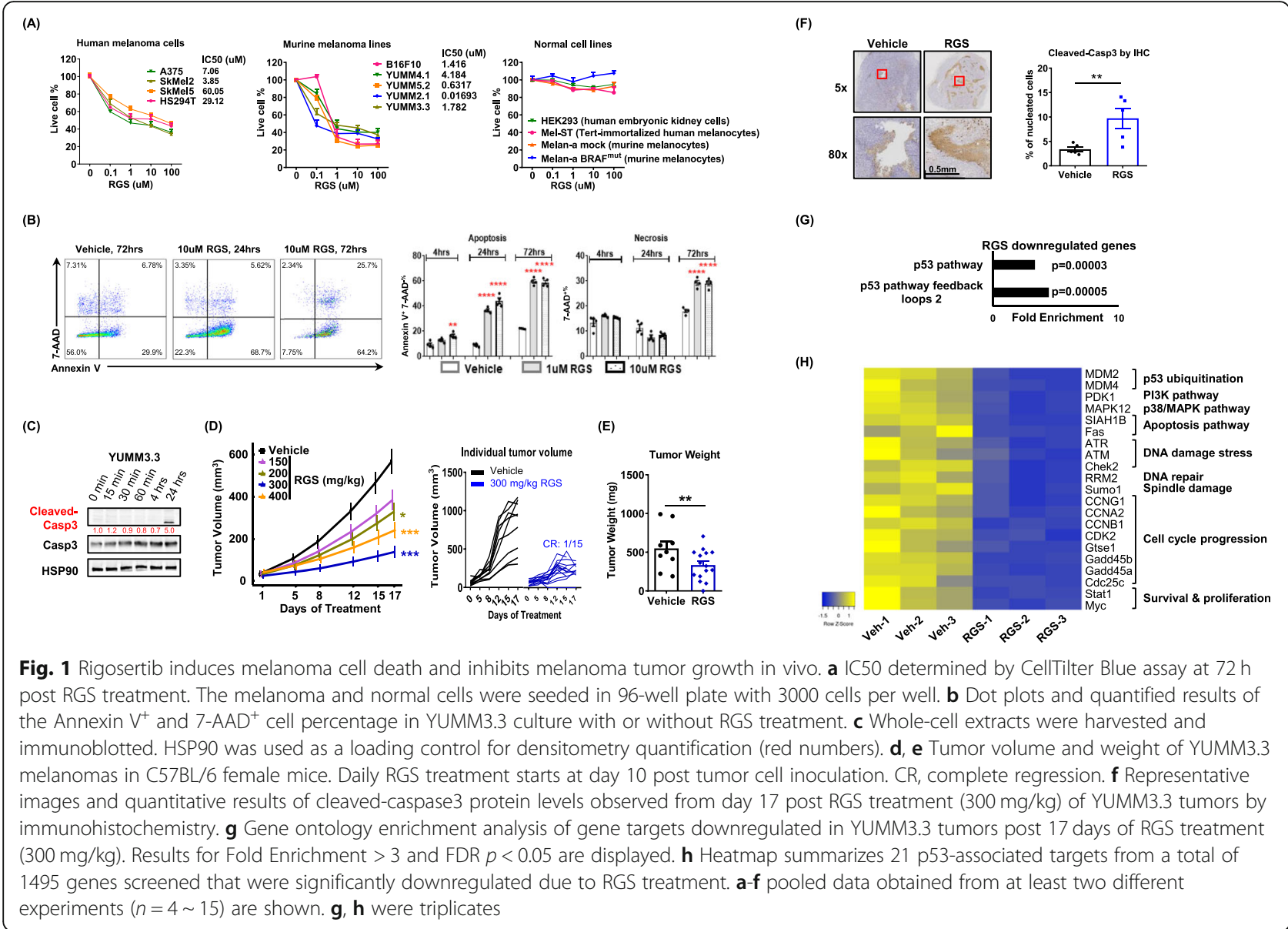

(Suppl.Fig. 1C). Instead, RGS treatment rapidly (< $15 \mathrm{mins}$ ) suppressed phosphorylation of $\mathrm{AKT}^{\mathrm{T} 308}$ in SkMel5 and YUMM3.3 melanoma cells and AKT ${ }^{\text {Ser473 }}$ in A375 and SkMel5 melanoma cells (Suppl.Fig. 1D). Consistent with the apoptosis assay results, there was a clear induction of cleaved-caspase3 in YUMM3.3 cells at $24 \mathrm{~h}$ post-RGS treatment (Fig. $1 \mathrm{c}$ ). In contrast, mitotic markers PLK1 and FOXM1 were rapidly and consistently reduced in SkMel5 melanoma cells after RGS treatment (Suppl.Fig. 1E). Together, these results demonstrate that RGS suppresses AKT activation, inhibits cell viability and promotes ICD (e.g., ROS production and apoptosis/necrosis) in melanoma cells.

To identify the optimal effective in vivo dose of RGS in the melanoma tumors, we conducted a doseescalation experiment $(0,150,200,300,400 \mathrm{mg} / \mathrm{kg})$ on YUMM3.3 tumors in C57BL/6 mice (Fig. 1 d). Oral delivery of RGS resulted in significant dose-dependent inhibition of tumor volume after 17 days of treatment that reached a plateau at 300 and $400 \mathrm{mg} / \mathrm{kg}$ doses with comparable $60 \%$ inhibition of tumor volume. We also observed that one of 15 mice exhibited complete tumor regression in response to the treatment (Fig. $1 \mathrm{~d}$ ).
Significant inhibition of tumor weight occurred with RGS treatment at $300 \mathrm{mg} / \mathrm{kg}$ dose (Fig. $1 \mathrm{e}$ ).

To test whether RGS-induced anti-tumor effects are $\mathrm{BRAF}^{\text {mut }}$-specific or gender-dependent, we administrated RGS to female mice bearing NRAS ${ }^{\mathrm{WT}} \mathrm{BRAF}^{\mathrm{WT}}$ B16F10 tumors (Suppl.Fig. 2A), as well as to male mice bearing YUMM3.3 tumors (Suppl.Fig. 2B). Up to $60 \%$ of tumor growth inhibition was observed in both models. Similar to the in vitro observations (Fig. 1 c), the levels of cleaved-caspase 3 were significantly increased in RGStreated YUMM3.3 tumor sections compared to the vehicle control-treated counterparts (Fig. $1 \mathrm{f}$ ). Notably, the YUMM3.3 murine melanoma with constitutively active BRAF (RAS binding independent) was sensitive to RGS treatment. This indicates that in $\mathrm{BRAF}^{\text {mut }}$ melanoma the RGS-induced inhibitory effects may not be dependent on RAS binding to activate RAF, highlighting a role for RGS in the inhibition of other RAS-downstream signals (such as PI3K/AKT) that are dependent on RAS binding to RAS-binding domain (RBD) for activation.

To provide a more comprehensive assessment of RGS responses, we performed bulk RNA sequencing analysis on the YUMM3.3 tumors (RGS and vehicle treated), 
including tumor cells, stromal cells, and immune cells. RGS specifically down-regulated genes are involved in the p53 pathway (Fig. $1 \mathrm{~g}$, h). Specifically, RGS treatment reduced the expression of genes encoding proteins involved in p53-ubiquitination, including MDM2 and $M D M 4$. In addition, GO Consortium pathway analysis indicated that these down-regulated genes function in the activation of PI3K-MAPK pathways, DNA damage/ repair, spindle damage, cell cycle progression, as well as survival and proliferation. The data revealed increased expression of genes involved in the plasminogen activating cascade and synaptic vesicle trafficking in RGStreated tumors (Suppl.Fig. 3). Query through the Vesiclepedia database (microvesicles.org) showed that the all of RGS-induced synaptic vesicle genes are encoding proteins that are located in either tumor microvesicles or exosomes. Interestingly, increased tumor vesicle formation has been linked to ICD [39].

\section{Rigosertib converts the immune environment of melanoma tumors from "cold" to "hot"}

Having demonstrated a role for RGS in promoting melanoma cell death and suppressing melanoma tumor growth in vivo, we next questioned how RGS might shape the immune system in the TME and TDLNs. Immune profiling by flow cytometric analysis showed that RGS treatment of mice bearing YUMM3.3 tumors promoted an increase in the total number and density of tumor-infiltrating leukocytes (Fig. 2 a). To identify the immune populations responsible for the anti-tumor effects, we first subtyped the intratumoral DCs that are likely to respond to the tumor antigens released as a result of the RGS-induced ICD (Fig. 2 b). We observed that there was a significant increase of the tumorinfiltrating DCs (Fig. 2 c), specifically CD $11 b^{-/ \text {low }}$ CD 11 $\mathrm{c}^{\text {low }}$ Ly6C $\mathrm{C}^{+}$plasmocytoid DCs (pDCs). Consistently, RGS also triggered the induction of $\mathrm{CD}_{11} \mathrm{~b}^{- \text {low }} \mathrm{DCs}$ in the TDLN (Fig. 2 c). In contrast to the TME profile where

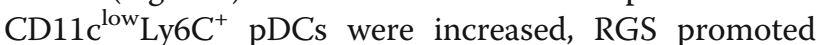
the frequency of $\mathrm{CD} 11 \mathrm{c}^{\mathrm{hi}} \mathrm{Ly} 6 \mathrm{C}^{-}$conventional DCs (cDCs) in the TDLNs. We observed that $\mathrm{CDC}$ possess > 2-fold the level of MHCII on the cell surface as compared to pDCs of melanoma-bearing mice (Fig. 2 d). This suggests that increased CDC numbers in TDLN of RGS-treated mice may facilitate improved tumor antigen presentation; thus, the DC subset-specific induction matches well with the tissue-specific immune functions. Given that both lymphoid tissue-resident $\mathrm{CD}^{+}$and $\mathrm{CD}_{103}{ }^{+}$immigrating DCs have excellent capacities to cross-present exogenous tumor antigens to activate $\mathrm{CD}^{+} \mathrm{T}$ cells [40], RGS treatment induced the frequency of these DC subsets in the TME and their levels are negatively correlated with tumor burden (Fig. 2 e).

Next, we profiled the immune effector cells in the TME. Within the lymphoid lineage, tSNE analysis showed that RGS induced a dose-dependent induction

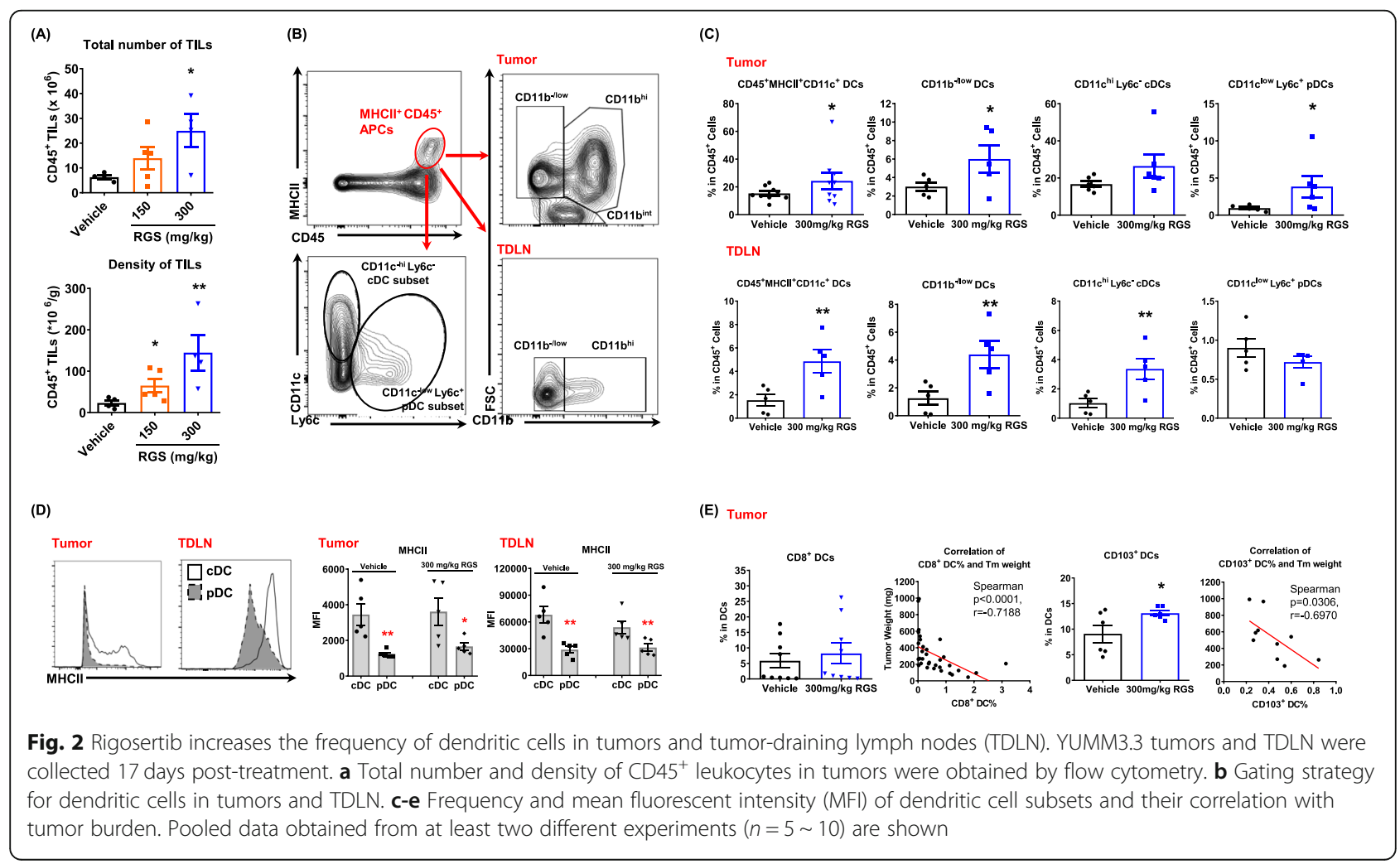


of $\mathrm{T}$ cells and NK cells while it reduced B cells in tumors (Fig. 3 a). We found that RGS increased not only the frequency of $\mathrm{CD}^{+} \mathrm{T}$ helper (Th) and $\mathrm{CD} 8^{+}$cytotoxic $\mathrm{T}$ cells (Tc) in the TME (Fig. 3 b, c), but also their activation, as reflected by elevated CD69 expression (Fig. $3 \mathrm{~d}$ ). The percentage of tumor-infiltrating $T$ cells was significantly and negatively correlated with the tumor burden (Spearman $p<0.0001, \mathrm{r}=-0.7924$ ) (Fig. $3 \mathrm{e}$ ), suggesting that RGS-induced $\mathrm{T}$ cell responses play an important role in tumor control. The increased $\mathrm{T}$ cell frequency was further confirmed by IHC staining of the tumor sections (Fig. $3 \mathrm{f}$ ) and TDLNs (Suppl.Fig. 4). While the $\mathrm{CD}^{+}$and $\mathrm{CD}^{+}$tumor infiltrate was increased in the RGS-treated tumors, there was no significant alteration in the level of $\mathrm{CD}^{+} \mathrm{FoxP}^{+} \mathrm{T}$ regulatory cells (Tregs) or angiogenesis (CD31) (Fig. $3 \mathrm{f}$ ). Notably, the percentages and activity of NK cells were increased in the RGStreated tumors (Fig. 3 g). While there was an induction of DC, T cell, and NK cell responses within the RGStreated tumors, we observed a $20 \%$ decrease of total $\mathrm{CD}_{11 \mathrm{~b}^{+}}$myeloid lineage populations (Fig. $3 \mathrm{~h}$, i). Among the specific myeloid populations, $\mathrm{F} 4 / 80^{+}$macrophages, especially the $\mathrm{CD}_{206^{+}}$M2-like macrophages, decreased most prominently in a dose-dependent manner in response to RGS treatment. While the level of $\mathrm{MHCII}^{+}$ M1-like macrophages did not correlate with the tumor burden, there was a significant and direct correlation between the decrease in $\mathrm{CD}_{206^{+}}$M2-like macrophages and tumor burden (Spearman $p<0.0001, \mathrm{r}=0.6400$ ). To determine whether Th and Tc cells are required for the anti-melanoma activity of RGS, we conducted depletion experiments of CD4 and/or CD8 cells (Fig. 3 j). Interestingly, depletion of CD8 cells alone, or depletion of both CD8 and CD4 cells, but not depletion of CD4 cells alone, significantly abrogated tumor growth inhibition by RGS treatment (Fig. $3 \mathrm{k}$ ). These results suggest that CD8 cells are the main population responsible and necessary for RGS anti-tumor activity. We further identified that RGS-induced caspase-dependent cell death is important to induce the anti-tumor responses in vivo since the pan-caspase inhibitor Z-VAD treatment blocked RGS-induced tumor growth inhibition and reduced $\mathrm{CD}^{+} \mathrm{T}$ cell infiltration (Suppl.Fig. 5).

The safety profile of RGS in tumor-free (Suppl.Fig. 6A, B) and tumor-bearing (Suppl.Fig. 6C) mice was examined to evaluate the safety of RGS as a therapy. RGS
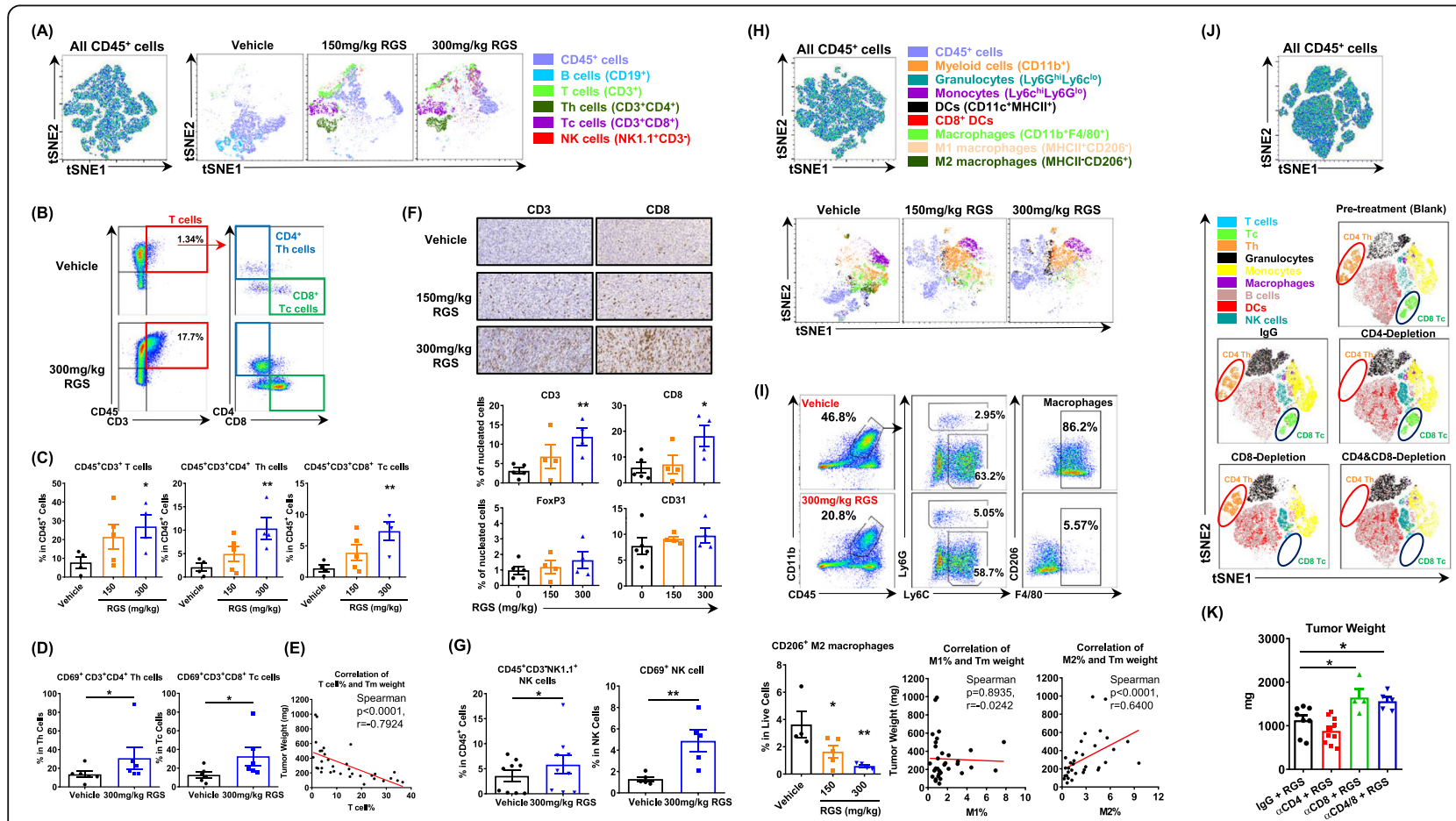

(K)

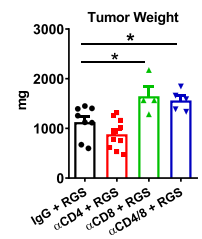

Fig. 3 Rigosertib promotes T cell and NK cell responses but attenuates tumor-associated M2 macrophages in the tumor microenvironment. YUMM3.3 tumors were collected 17 days post-treatment. a, h Live CD45 leukocytes were concatenated after downsampling to 20,000 events for subsequent high-dimensional data analysis to normalize the contribution among samples under different treatments. Samples were then analyzed in parallel by t-SNE and manually gated leukocyte populations were overlaid onto the total t-SNE map using FlowJo 10.5.3. b-e, g, i Flow cytometric and $\mathbf{f} \mathrm{IHC}$ analysis of YUMM3.3 tumors at day 17 post-treatment. $\mathbf{j}$ Flow cytometric tSNE analysis of PBMC samples from YUMM3.3 tumor-bearing mice treated with RGS before and after CD4 and/or CD8 antibody depletion treatments. (K) Tumor weight (day 16 post RGS treatment) of YUMM3.3 tumors in C57BL/6 mice with and without CD4 and/or CD8 depletion. a-i pooled data obtained from at least two different experiments ( $n=5 \sim 10$ ) are shown. j, k were replicates ( $n=10$ per group) 
treatment did not induce severe toxicity in mice, as there was no significant loss of body weight or increase in the levels of liver enzymes alanine aminotransferase (ALT) and aspartate aminotransferase (AST) in the serum of RGS treated as compared to the vehicle- treated mice. Given that in vitro treatment with RGS inhibited the mitosis/proliferation and promoted ICD of melanoma cells (Fig. 1 b, e \& Suppl.Fig. 1), RGS's impact on the cellular turnover of normal tissues (Suppl.Fig. 6B) was studied. No alteration of Ki67 levels was observed in the intestine, spleen, and skin tissue after 15 days of RGS treatment.

\section{Rigosertib improves responses of aPD-1/aCTLA-4 therapies}

Since RGS treatment elicits an anti-tumor immune activation profile, we postulated that the addition of RGS to $\alpha \mathrm{PD}-1 / \alpha \mathrm{CTLA}-4$ would improve therapeutic outcomes of melanoma tumors with poor response to ICB. The B16F10 model is reported to be highly aggressive and non-responsive to ICB therapies (such as $\alpha \mathrm{PD}-1$, $\alpha \mathrm{PD}$ -
L1, $\alpha$ CTLA- 4 or a combination of $\alpha$ CTLA- 4 with either $\alpha \mathrm{PD}-1$ or $\alpha \mathrm{PD}-\mathrm{L} 1)$ [41], which makes it ideal to model clinical responses of melanomas that are either nonresponsive to, or have progressed on, immune checkpoint inhibitors. Therefore, we pre-treated BRAF ${ }^{\mathrm{WT}}$ /NRAS ${ }^{\mathrm{WT}}$ B16F10 tumors with 3 doses of $\alpha \mathrm{PD}-1$ to validate the ICB non-responsive phenotype and then administrated either vehicle + IgG control, RGS + IgG, vehicle $+\alpha$ PD- $1 / \alpha$ CTLA- 4, RGS $+\alpha$ PD- $1 / \alpha C T L A-4$, or dabrafenib (BRAF inhibitor) + trametinib (MEK inhibitor) $+\alpha$ PD $-1 / \alpha$ CTLA- 4 as a control of clinically used combinatorial therapy (Fig. 4a). As expected, $\alpha \mathrm{PD}-1 /$ $\alpha$ CTLA- 4 alone or in combination with dabrafenib + trametinib did not exhibit a beneficial effect on mouse survival (Fig. 4 b) or tumor growth inhibition (Fig. 4 c, d). In contrast, the combination of RGS plus $\alpha \mathrm{PD}-1 /$ $\alpha C T L A-4$ improved mouse median survival to 22.5 days compared to 11 days in the vehicle + IgG control or monotherapy groups (Fig. 4 b) and resulted in $~ 70 \%$ inhibition of tumor growth (Fig. $4 \mathrm{c}$, d). The effects of RGS plus $\alpha$ PD- $1 / \alpha$ CTLA- 4 treatments were synergistic,

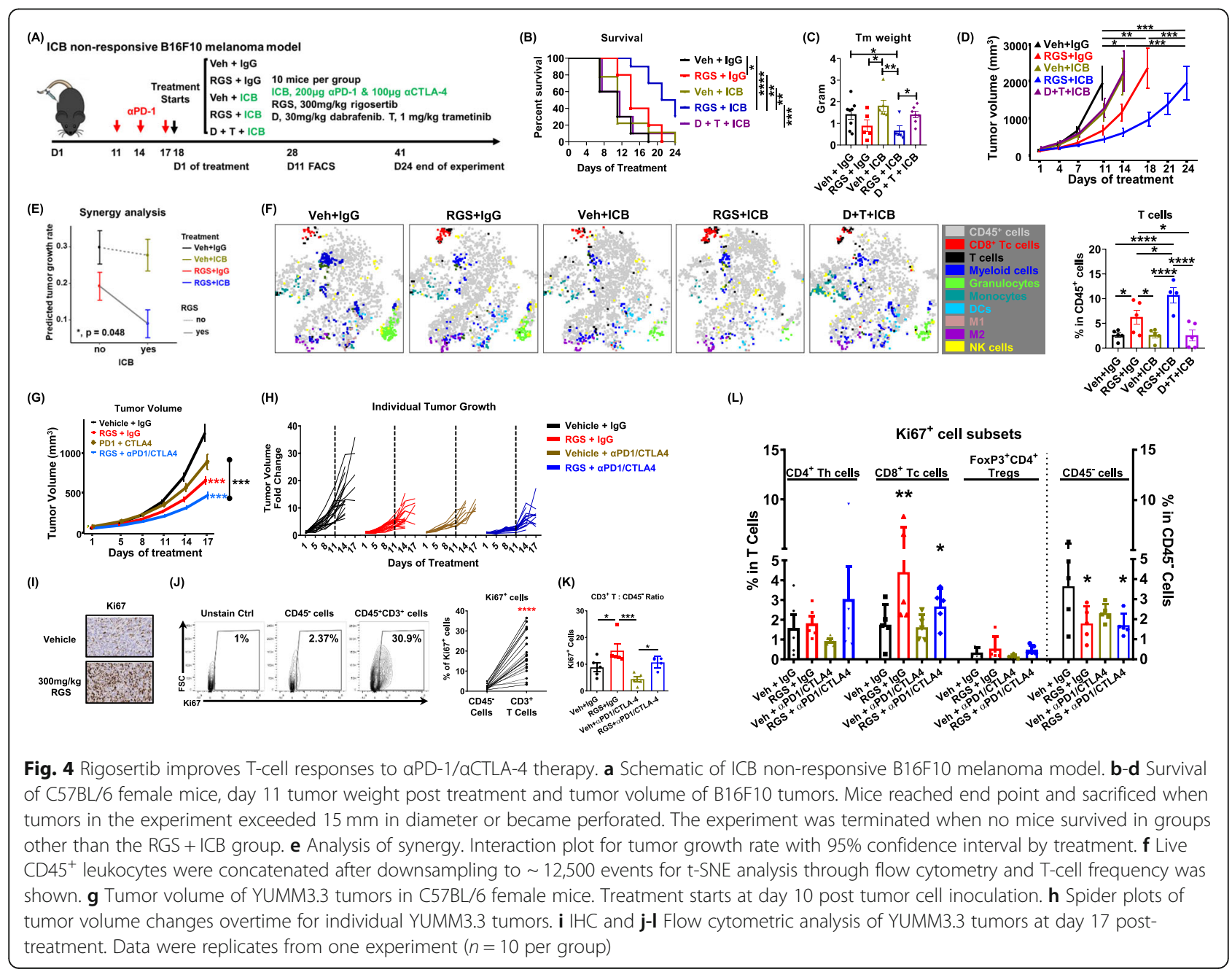


based on a plot of interaction effect on tumor growth rate estimated by the mixed-effect model (Fig. 4 e). Detailed immune profiling of tumor-infiltrating leukocytes through tSNE analysis showed an improved T-cell response, especially $\mathrm{CD}^{+}{ }^{+}$Tc cells, in tumors treated with either RGS monotherapy or with RGS in combination with $\alpha$ PD- $1 / \alpha$ CTLA-4 (Fig. $4 \mathrm{f}$ ). The frequency of T cells within the TME increased from $2.6 \pm 0.5 \%$ in the control group to $6.3 \pm 1.5 \%$ with RGS treatment alone, and reached to $10.7 \pm 1.5 \%$ in the RGS plus $\alpha$ PD- $1 / \alpha$ CTLA- 4 treatment group (Fig. $4 \mathrm{f}$ ).

We next validated these results using BRAF $^{\text {mut }}$ YUMM3.3 model. While $\alpha$ PD-1/ $\alpha$ CTLA- 4 therapy alone exhibited marginal growth inhibition of the YUMM3.3 tumors after 17 days of treatment, the addition of RGS to the $\alpha \mathrm{PD}-1 / \alpha C$ TLA- 4 regimen resulted in a significantly greater reduction in tumor volume (70\%) (Fig. 4 g) as compared to RGS alone. While $>90 \%$ of tumors receiving either monotherapy (RGS or ICB) exhibited a rapid growth at 11 days post-treatment compared to baseline size, tumors under the combinatorial treatment grew a bit more slowly the first 11 days after treatment initiation (Fig. $4 \mathrm{~h}$ ). Interestingly, there was a significant increase in $\mathrm{Ki} 67^{+}$cells in the tumors receiving either RGS or $\alpha$ PD- $1 / \alpha C$ TLA- 4 or both (Fig. 4 i). Flow cytometric analysis was performed to identify specific cell populations with high Ki67 expression. Analysis of the Ki67 ${ }^{+}$cells revealed that only $2.37 \%$ of the $\mathrm{CD} 45^{-}$cells, which includes tumor cells, were $\mathrm{Ki}^{+} 7^{+}$(Fig. 4 j). In sharp contrast, $>30 \%$ of $\mathrm{CD}_{4} 5^{+} \mathrm{CD}^{+} \mathrm{T}$ cells were $\mathrm{Ki} 67^{+}$, indicating that these $\mathrm{T}$ cells are proliferating in melanoma tumors. Notably, both RGS monotherapy and combinatorial treatments of RGS + $\alpha$ PD $-1 / \alpha C$ CLA -4 increased the ratio of proliferating Ki67 ${ }^{+}$T-cell to $\mathrm{CD} 45^{-}$cells (Fig. 4 k). Further subtyping analysis suggested that RGS and combined RGS $+\alpha P D-1 /$ $\alpha C T L A-4$ treatments specifically promoted proliferation of $\mathrm{CD}^{+}$Tc cells, but not $\mathrm{CD} 4^{+}$Th cells nor the FoxP3 ${ }^{+}$ Tregs while attenuating the proliferation of $\mathrm{CD} 45^{-}$cells in the TME (Fig. $4 \mathrm{l}$ ). Consistent with the immune profile in the TME, increased frequency and activation of NK cells, $\mathrm{CD}^{+}$Th and $\mathrm{CD}^{+}$Tc cells were also identified in the TDLN of the combinatorial treatment group (Suppl.Fig. 7). Notably, while $\alpha$ PD-1/ $\alpha$ CTLA- 4 treated tumors did not exhibit an elevated effector cell response at day 17 after treatment initiation, there was a clear induction of NK and $\mathrm{CD}^{+}{ }^{+}$Th cell activities, as well as $\mathrm{CD}^{+}{ }^{+} \mathrm{Tc}$ cell frequency, in the TDLN. The RGS plus ICB treatment did not result in significant loss of mouse body weight and did not increase spleen weight or serum levels of liver enzymes ALT and AST, as compared to the vehicle group (Suppl.Fig. 8).

To explore the cytokine production [IFN- $\gamma$, TNF- $\alpha$ and granzyme $\mathrm{B}(\mathrm{GzmB})]$ and antigen (Ag)-specificity of treatment-induced $\mathrm{CD}^{+}$Tc responses in the tumor microenvironment, we conducted in vitro recall experiments using the tumor-infiltrating T-cell samples isolated from above YUMM3.3 and B16F10 tumors (Suppl.Fig. 9). PBMCs isolated from tumor-free C57BL/6 mice were used as a negative control for antigen specificity. The stimulation with $\alpha \mathrm{CD} 3 / \mathrm{CD} 28$ beads was used as a positive control for $\mathrm{CD}^{+}$Tc cell responses. The production of TNF- $\alpha$ in tumor-infiltrating Tc cells was not detected in any tumor samples. IFN- $\gamma$ in tumorinfiltrating Tc cells from B16F10 tumors was not detected. As expected, the positive control of $\alpha \mathrm{CD} 3 / \mathrm{CD} 28$ beads stimulated IFN- $\gamma$ production in $>80 \%$ of Tc cells isolated from the tumor-free peripheral blood samples (Ag-naive PBMC). The autologous antigen stimulation from parental YUMM3.3 tumor irradiated cells significantly induced IFN $-\gamma$ production in $\sim 20 \%$ of Tc cells isolated from tumors undergo RGS + ICB treatment, compared to either Ag-naive PBMC or Tc cells isolated from tumors that were treated with vehicle+IgG. Interestingly, $\alpha \mathrm{CD} 3 / \mathrm{CD} 28$ beads did not stimulate $\mathrm{GzmB}^{+} \mathrm{Tc}$ cells in the PBMC samples (stimulated for $4 \mathrm{~h}$ at $37^{\circ} \mathrm{C}$ and $4{ }^{\circ} \mathrm{C}$ overnight). This observation is consistent with the report that the induction of GzmB-containing cytotoxic granules in $\mathrm{T}$ cells using stimulation with $\alpha \mathrm{CD} 3 /$ $\mathrm{CD} 28$ beads, required at least 3 days, occurred after several rounds of cell division, and required cell cycle progression [42]. Nevertheless, cells isolated from tumors treated with RGS + IgG exhibited an increase of $\mathrm{GzmB}^{+}$ Tc cells in both B16F10 and YUMM3.3 tumors. In the RGS + IgG group, there was a significant induction of $\mathrm{GzmB}^{+}$Tc cells with stimulation of irradiated YUMM3.3 cells compared to Ag-naive PBMC samples, which is comparable to the level in the positive control of $\alpha \mathrm{CD} 3 /$ CD28 beads, suggesting that the RGS-induced Tc cell response is at least partially antigen-specific.

The programmed death-ligand 1 (PD-L1)/PD-L2/PD-1 axis delivers inhibitory signals that function as a brake for immune responses in cancer [43]. Recently, PD- $\mathrm{L}^{+}$ $B$ cells were identified to be a feature of regulatory $B$ cells (Bregs) that are critical regulators of anti-cancer immunity [44, 45]. We found that RGS and/or ICB $(\alpha \mathrm{PD}-1 / \alpha \mathrm{CTLA}-4)$ treatments did not alter $\mathrm{B}$ cell generation in the bone marrow (Suppl.Fig. 10A). Instead, there was a significant reduction of B cells in the tumor, TDLN, and spleen tissues under the RGS + ICB treatment. The reduction of tumor-infiltrating $B$ cells was confirmed by IHC staining (Suppl.Fig. 10B). While all the tumor-infiltrating $\mathrm{B}$ cells were $\mathrm{PD}-\mathrm{L}^{+}{ }^{+}$Bregs, some of them were co-expressing $\mathrm{PD}-\mathrm{L}_{2}{ }^{+}$and belong to the antibody-producing follicular B cell (FOB) lineage (Suppl.Fig. 10C). Detailed profiling of the B cell subsets in the TME [46] suggested that while the RGS + ICB treatment attenuated the $\mathrm{FOB}$ population, it promoted the CD5 $^{-}$B1b cells (Suppl.Fig. 10D,E). Though there is very 
limited functional characterization of how these B cell subsets may perform in tumor immunity in the literature, B1b cells were reported to confer $\mathrm{T}$ cellindependent long-lasting protective immunity against infections [47].

\section{Rigosertib induces CD40 on melanoma cells and fuels the anti-tumor immunity}

In order to gain further insight into molecular mechanisms of anti-tumor activity of RGS and RGS + ICB combined treatments, tumor cytokines in drug-treated versus vehicle-treated tumor-bearing mice were examined using a cytokine array (Fig. 5 a, b). We observed that CD40 was increased 30-fold in tumors from mice that received either RGS monotherapy or the RGS + $\alpha \mathrm{PD}-1 / \alpha \mathrm{CTLA}-4$ combined treatment as compared to control vehicle-treated mice. While both antigenpresenting cells (APCs, including DCs, macrophages and $B$ cells) and melanoma cells were reported to express CD40 [26, 27], subsequent tSNE analysis by flow cytometry showed that in our experimental setting RGS treatment-induced CD40 expression on $\mathrm{CD} 45^{-}$cells but not on APCs (Fig. 5 c). To identify the origin of these $\mathrm{CD} 40^{+} \mathrm{CD} 45^{-}$cells, we inoculated YUMM3.3 melanoma cells into mice harboring the tdTomato, which was inserted into the Gt (ROSA)26Sor locus (ROSA mice) so that every single cell originating from these mice would exhibit tdTomato ${ }^{+}$in flow cytometric analysis [48]. As expected, $\sim 93.6 \%$ of the $\mathrm{CD}_{4} 5^{+}$leukocytes were tdTomato $^{+}$, confirming that they originate from ROSA mice (Fig. 5 d). We observed that $~ 91.3 \%$ of the RGS-induced $\mathrm{CD} 40^{+} \mathrm{CD} 45^{-}$cells did not express tdTomato (tdTomato ${ }^{-}$), suggesting that they were tumor cells that were injected into mice to generate tumors rather than the endogenous stromal cells from ROSA mice. Of interest, these $\mathrm{CD} 40^{+} \mathrm{CD} 45^{-}$cells in RGS-treated mice exhibited increased expression of co-stimulatory molecules CD80 and ICOS-L, with a baseline level of MHC-I and unaltered PD-L1 expression (Fig. 5 e). Costimulatory molecules, such as CD40, CD80 and inducible T-cell costimulator ligand (ICOS-L), all provide signals for activating cell-mediated immune responses $[49,50]$, further indicating that RGS treatment is inducing an immune response.

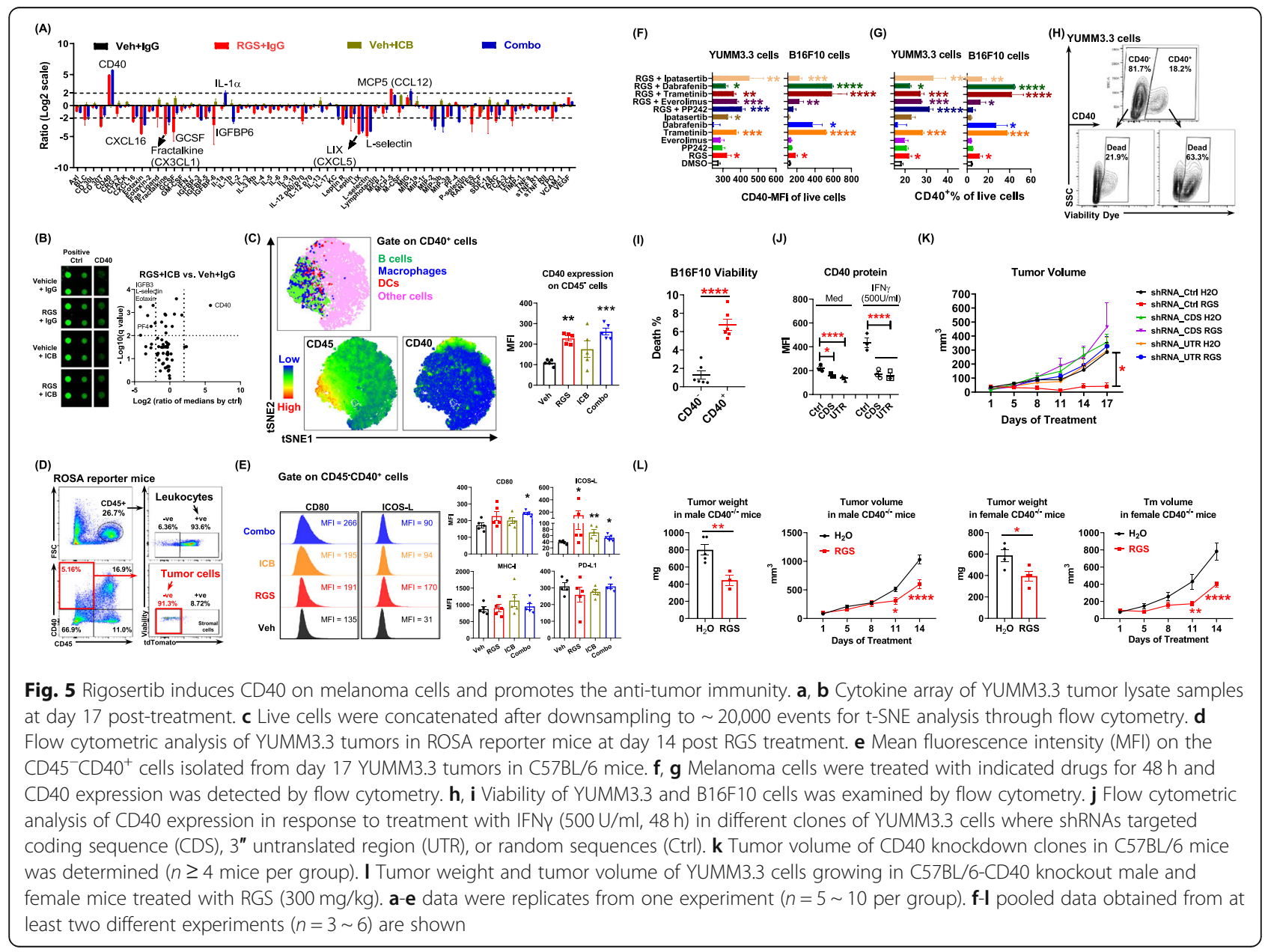


As a RAS mimetic, RGS has the potential to block oncogenic RAS-RAF-MEK-ERK and/or PI3K-AKTmTOR signaling pathways [15-17]. To explore whether RGS could directly induce CD40 expression in melanoma cells, and if so, which RAS-mediated pathway(s) may be responsible for the phenotype, we evaluated the impact of serval pathway specific inhibitors on CD40 plasma membrane expression in melanoma cells in vitro, including mTOR kinase inhibitor PP242 $(5 \mu \mathrm{M})$, mTORC1 inhibitor Everolimus (10 nM), MEK inhibitor Trametinib $(0.1 \mu \mathrm{M})$, BRAF inhibitor Dabrafenib $(1 \mu \mathrm{M})$, AKT inhibitor Ipatasertib $(10 \mu \mathrm{M})$, and/or RGS $(0.1 \mu \mathrm{M})$. We observed that RGS directly induced CD40 intensity (Fig. $5 \mathrm{f}$ ) and the frequency of CD40-membrane positive cells (Fig. 5 g) in B16F10 and YUMM3.3 cells. While mTOR inhibition (PP242 and Everolimus) did not promote CD40 expression on melanoma cells, suppression of either MEK or AKT pathways, induced CD40 expression in BRAF ${ }^{\text {mut }}$ YUMM3.3 cells. The inhibition of MEK or BRAF in BRAF ${ }^{\mathrm{WT}}$ B16F10 cells resulted in a $\sim 10$-fold increase of CD40 expression. Notably, when RGS was combined with any of these RAS pathway inhibitors, the induction of CD40 expression in melanoma cells was further increased, suggesting pathways downstream of RAS may cooperate to restrain baseline CD40 expression in melanoma cells. It has been reported that CD40 signaling induces malignant cell death in many cancer types, including renal cell carcinoma [29], urothelial cell carcinoma [30], ovarian carcinoma [31, 32], cervical carcinoma [33] and bladder carcinoma [34]. Here, we identified that the death rate of $\mathrm{CD} 40^{+}$ melanoma cells was 3 -fold higher than in $\mathrm{CD} 40^{-}$melanoma cells for both RGS-treated YUMM3.3 (Fig. 5 h) and B16F10 models (Fig. 5 i).

To determine whether induction of CD40 expression was required for melanoma tumor cell response to RGS, we next knocked down the expression of CD40 in YUMM3.3 melanoma cells (CD40KD) by targeting either the coding sequence (CDS) or the 3 ' untranslated region (UTR) to achieve $\sim 60 \%$ knockdown of CD40 mRNA (Suppl.Fig. 11) and protein expression (Fig. 5 j). The CD40KD melanoma cells were resistant to IFNYmediated induction of CD40 expression (Fig. 5 j). Furthermore, while shRNA control vector transfection responded to RGS treatment with reduced YUMM3.3 tumor growth in vivo, tumors with attenuated CD40 expression (shRNA_CDS and shRNA_UTR) were resistant to RGS-mediated inhibition of tumor growth (Fig. $5 \mathrm{k}$ ). These data illustrate a requirement of melanoma cell expression of CD40 for RGS-mediated tumor growth control. To evaluate the importance of host-derived CD40 in RGS-induced responses, we injected YUMM3.3 cells into CD40 knockout (CD40KO) mice (Fig. 5 l). Notably, YUMM3.3 tumors responded to RGS treatment in
CD40KO mice, however the response was not as prominent as in CD40 wild-type mice ( $30 \sim 45 \%$ growth inhibition post-two-weeks of treatment vs. $50 \%$, respectively). These results suggest that melanoma cellintrinsic CD40, rather than host-derived CD40, plays a major role in the RGS-mediated inhibition of tumor growth. In addition, we explored the CD40 ligand (CD40L) expression on T cells in the tumor microenvironment (Suppl.Fig. 12) and found that $~ 50 \%$ and $~ 10 \%$ of $\mathrm{CD}^{+}{ }^{+}$Th cells express CD40L in the YUMM3.3 and B16F10 tumors, respectively. Notably, RGS + ICB significantly induced the frequency of $\mathrm{CD}_{40 \mathrm{~L}^{+}} \mathrm{Th}$ cells in the tumor microenvironment. Compared to the Th cells, $\mathrm{CD}^{+}$Tc cells exhibited a more abundant CD40L expression with Tc cells from YUMM 3.3 cells being $90 \% \mathrm{CD} 40 \mathrm{~L}^{+}$and from B16F10 tumors being $~ 40 \%$ $\mathrm{CD}_{40 \mathrm{~L}^{+}}$. The availability of CD40L on tumor-infiltrating $\mathrm{T}$ cells support our proposed model of CD40-dependent and Tc-dependent immunogenic cell death of tumor cells. Of note, $\mathrm{CD} 4^{+}$Th cells from tumors treated with RGS exhibited a 50\% increase in CD40L expression, while $\mathrm{CD}^{+}$Th cells from RGS + ICB treated tumors exhibited only about a $15 \%$ increase in CD40L expression in YUMM3.3 tumors. In contrast, B16F10 tumors treated with RGS + ICB exhibited the greatest increase in CD40L expression. Collectively, our data indicate that the inhibition of RAS/RAF/PI3K-mediated pathways, as observed with RGS treatment, directly induce CD40 expression in melanoma cells to trigger immunogenic cell death, which is associated with increased expression of co-stimulatory signals (e.g., CD40, CD80, and ICOS-L) for mediating anti-tumor responses.

\section{Rigosertib and BRAF/MEK inhibitors induce CD40} expression in responsive patient melanoma cells in vivo To determine whether these findings regarding induction of CD40 expression observed in mouse melanoma models are recapitulated in human melanoma, we evaluated the effects of RGS on the growth of two human melanoma PDX tumors previously established and characterized in our laboratory [51]. PDX1179 had an $\mathrm{NRAS}^{\mathrm{Q} 61 \mathrm{H}}$ mutation and PDX1214 had NRAS ${ }^{\mathrm{Q} 61 \mathrm{~L}}$ and TP53 mutations (Suppl.Table 1). When these PDX tumors were implanted into NGS mice and evaluated for response to RGS as compared to vehicle, PDX1179 was completely resistant to RGS treatment, while PDX1214 exhibited $\sim 25 \%$ growth inhibition over 3 weeks of treatment (Fig. 6 a). Notably, we observed a significant increase in the levels of cleaved-caspase 3 and CD40 in PDX1214, but not in PDX1179. As expected, the major cellular population within these PDX tumors was $\mathrm{SOX}^{+} 0^{+}$melanoma cells. Notably, in PDX1214 the SOX10 expression pattern matched well with the CD40 expression pattern based upon IHC staining, even 


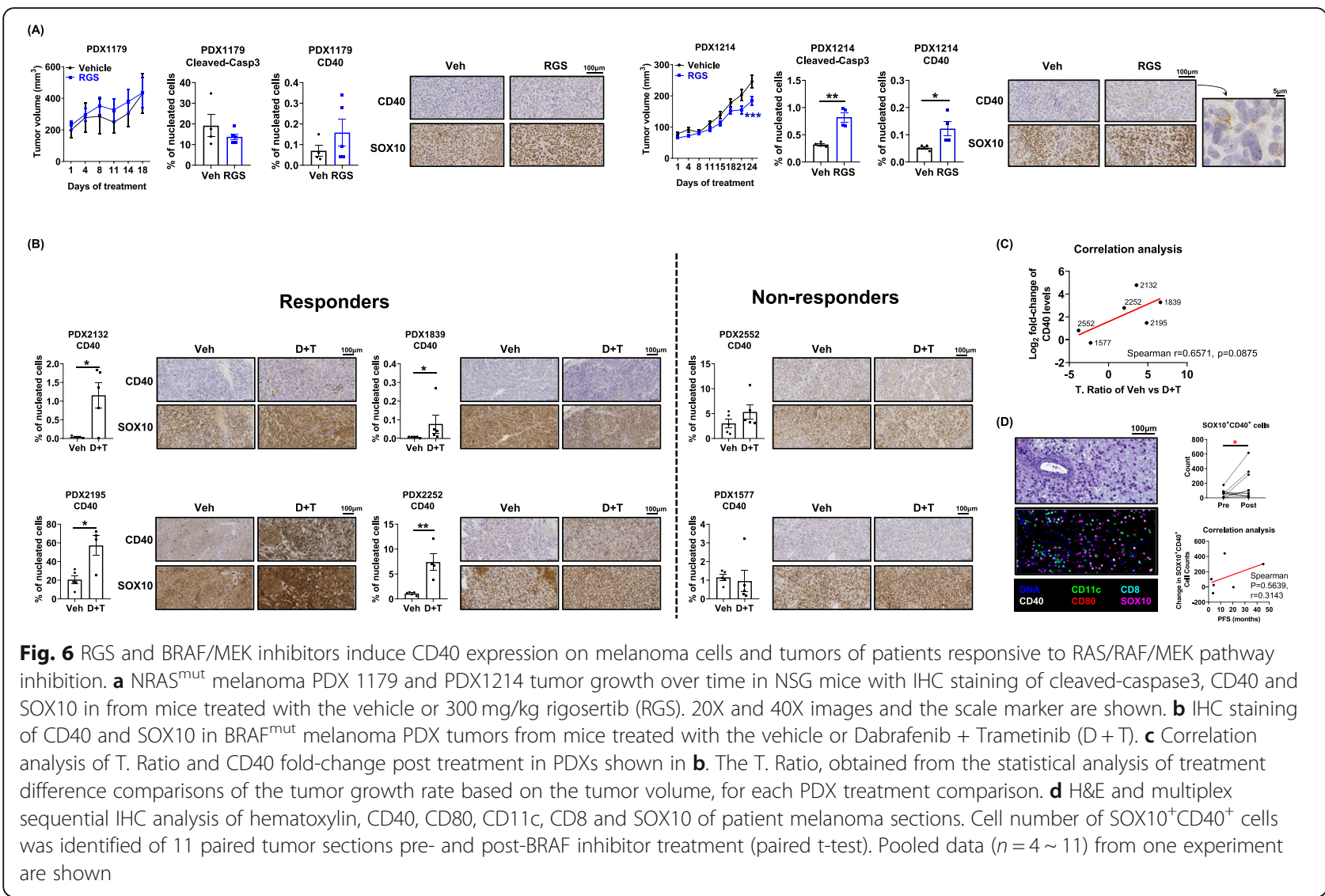

though there was only minimal inhibition of tumor growth. Since NGS mice lack a functional adaptive immune system, it is expected that any anti-tumor effects of RGS would be largely driven by the direct effect on PDX growth. Indeed, similar findings were observed of murine melanoma tumors (YUMM3.3 and B16F10) grown in athymic mice that do not have mature $\mathrm{T}$ cells, which were completely resistant to RGS (Suppl.Fig. 13), suggesting that the adaptive immune system is indispensable for the RGS-induced anti-tumor effects.

Since dabrafenib and trametinib also induce CD40 expression on melanoma tumor cells, we further examined response to this therapeutic combination $(30 \mathrm{mg} / \mathrm{kg}$ dabrafenib $+1 \mathrm{mg} / \mathrm{kg}$ trametinib, $\mathrm{D}+\mathrm{T}$ ) in mice harboring mutated $\mathrm{BRAF}^{\mathrm{V} 600}$ patient-derived tumors (Fig. $6 \mathrm{~b}$ ). Based on our published work [51], PDXs can be stratified into 2 distinct categories based of their sensitivity to D + T: responders (PDX2132, PDX1839, PDX2195 and PDX2252) and non-responders (PDX2552 and PDX1577). At the baseline level, CD40 expression varied among these PDXs ranging from 0.1 to $20 \%$ of all nucleated cells. Analysis of CD40 and SOX10 expression using IHC staining in $\mathrm{BRAF}^{\mathrm{V} 600}$ PDXs consistently showed a significant 3- to 15-fold induction of CD40 expression in all 4 responsive tumors compared to the respective vehicle control treated tumors. This was not the case in non-responsive tumors. To facilitate comparisons among PDX tumors and the responses to therapy, Tratios for treatment group comparisons across these $\mathrm{BRAF}^{\mathrm{V} 600}$ PDX tumors were generated (Fig. 6 c). The Tratio is a ratio of the difference in the slope of tumor growth between control and a treatment group relative to its $\mathrm{SE}$, calculated by estimated marginal means based on the mixed-effect model. We found a clear trend towards a positive correlation between the T-ratios of drug responsiveness and the fold increase of CD40 levels post $\mathrm{D}+\mathrm{T}$ treatment (Spearman $\mathrm{r}=0.6571, p=0.0875$ ).

We next conducted 6-color (hematoxylin, CD40, CD80, CD11c, CD8 and SOX10) multiplex IHC staining on the section of tumors from melanoma patients treated with BRAF inhibitor (Fig. 6 d). As expected, we observed $\mathrm{CD}_{40} 0^{+} \mathrm{SOX} 10^{+}$melanoma cells in the TME of all 11 post-treatment samples and in 10 of 11 pretreatment samples. The $\mathrm{CD} 40^{+} \mathrm{SOX} 10^{+}$cells in each tumor biopsy ranged from 2 to 616 counts. The number of $\mathrm{CD} 40^{+} \mathrm{SOX} 10^{+}$melanoma cells increased to 103 post BRAF treatment in the one tumor that did not have $\mathrm{CD} 40^{+} \mathrm{SOX} 10^{+}$cells prior to BRAF inhibitor treatment. Notably, we observed a significant induction of $\mathrm{CD} 40^{+} \mathrm{SOX} 10^{+}$cells in the tumors when pre-BRAF inhibitor treatment (mean counts $=57$ ) was compared to post-BRAF inhibitor treatment tissue (mean counts = 
154). Although we did not detect a statistically significant correlation between the change in the number of $\mathrm{CD}_{4} 0^{+} \mathrm{SOX} 10^{+}$cells in the TME post-treatment and the progression free survival (PFS) due to the small sample size and complications of prior treatments, we observed a clear trend for a positive correlation for $\mathrm{D}+\mathrm{T}$ induction of $\mathrm{CD}_{40} 0^{+} \mathrm{SOX} 10^{+}$cells for the 6 melanoma patients studied who had not undergone any treatment prior to BRAF/MEK inhibitor therapy (Spearman $r=0.3143$ ). Together, these data suggest that CD40 is induced in tumor cells of melanoma patients responding to the therapies targeting RAS/RAF/MEK pathway.

\section{Correlation of CD40 in survival and response to therapies in melanoma}

We next investigated whether CD40, CD80, and ICOS-L expression correlates with the outcome and therapeutic response in melanoma patients. By querying The Cancer Genome Atlas (TCGA, $N=480$ ) patient datasets of melanoma samples, we found that the $\mathrm{CD} 40$ expression was significantly correlated with CD80 (Spearman $p<0.0001$, $\mathrm{r}=0.6456)$ and ICOS-L (Spearman $p<0.0001, \quad \mathrm{r}=$ 0.5209 ) in tumors from melanoma patients (Fig. 7 a). Given that RGS treatment in mice increased CD40, CD80, and ICOS-L expression levels on tumor cells, and this was associated with a better therapeutic outcome, we questioned whether the levels of CD40/CD80/ICOS$\mathrm{L}$ have any prognostic value in melanoma patients. While the loss of CD40 copy number is very rare in melanoma patients $(\sim 1.25 \%$, TCGA), $\sim 50 \%$ of melanoma patients exhibit CD40 copy number gains (Fig. 7 b) and thus may be prone to exhibit enhanced inducible expression of CD40. Though the increased copy number of either CD40, CD80 or ICOS-L alone did not correlate with any changes in patient overall survival (OS), the triple-gain group of patients $(n=20)$ who exhibited increased copy number of CD40, CD80, and ICOS-L, exhibited a significantly better 10-year OS $(p=0.0326)$ (Fig. 7 b). This triple-gain group of patients did not exhibit a higher mutational burden (Fig. 7 b). Consistently, a correlation matrix profiling of gene mRNA expression in TCGA collection of melanoma tumors showed that CD40 expression significantly and positively correlates with CD4 and CD8 infiltrates, as well as with type 1 cytokine responses based on IFNY and GzmB expression patterns (Fig. 7 c). In addition, increased $\mathrm{T}$ cell responses may be a result of elevated $\mathrm{T}$ cell recruitment (based on association with chemokine CXCL9 implicated in $\mathrm{T}$ cell trafficking) and to a lesser extent, $\mathrm{T}$ cell proliferation (IL-2). Notably, type 2 cytokine responses (IL-5) and neutrophil responses (CXCL8/IL-8) were not altered by the changes of CD40 expression, suggesting

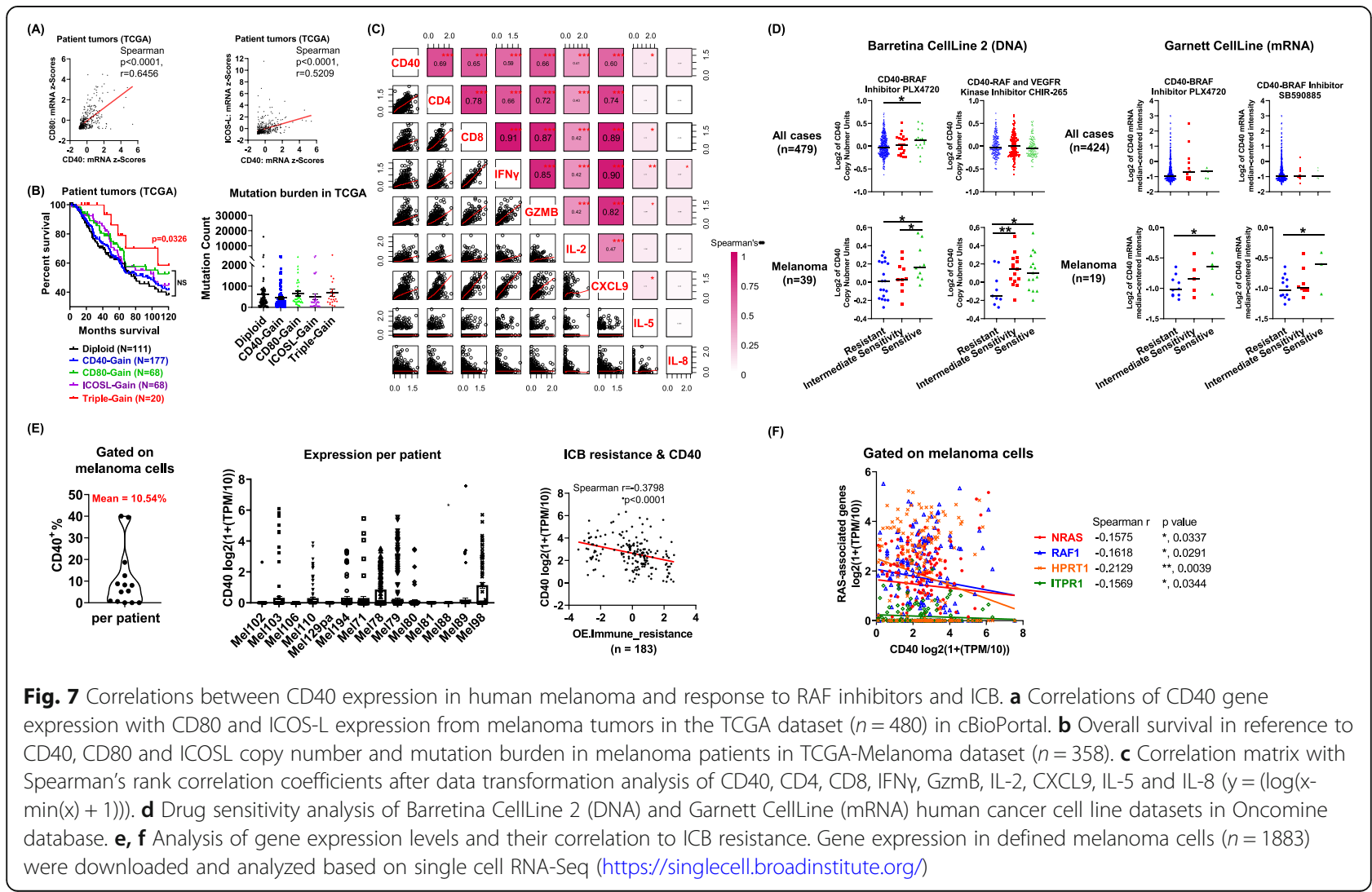


that CD40 expression specifically correlates to the Tcell-dependent antitumor immunity in human melanoma tumors.

Given that RGS interferes with RAS-RAF activation and triggers CD40-dependent anti-tumor effects in melanoma, we questioned whether the CD40 level is prognostic of response to previously used effective therapy, such as RAF inhibitor treatment. Thus, we further conducted drug sensitivity analysis of Barretina Cell Line 2 (DNA) and Garnett Cell Line (mRNA) datasets that are available in the Oncomine database (Fig. 7 d \& Suppl.Fig. 14). While the CD40 level does not correlate with the drug sensitivity to RAF inhibitors for all cancer types, human melanoma cells with increased CD40 at either copy number or mRNA levels are significantly more sensitive to the RAF inhibitor treatments. In contrast, CD80 and ICOSL are not correlated to the sensitivity of melanoma cells to the BRAF inhibitors (Suppl.Fig. 15).

Given the immune-modulatory role of CD40 to promote $\mathrm{DC}$ and $\mathrm{T}$-cell responses, we next questioned whether expression of CD40 would correlate with response to ICB immune therapy in melanoma. To do so, we analyzed the bulk tumor RNA-Seq results of the two largest available melanoma datasets with both transcriptomics and ICB immunotherapy response [52, 53]. We found that CD40 expression is significantly higher in tumors from melanoma patients that respond to ICB compared to the non-responsive patients (Suppl.Fig. 16). To further dissect the prognostic value of melanoma cell-intrinsic CD40 to ICB, we retrieved and analyzed the single cell (sc) RNA-Seq dataset of melanoma patients from Single Cell Portal (Broad Institute) [54]. This dataset contains gene expression and ICB response profiles from 14 melanoma patients from whom 1883 melanoma cells were sc-sequenced (Fig. 7 e). We determined that the mean frequency of $\mathrm{CD} 40$ positive melanoma cells in these patients is $10.54 \%$. There is a wide range of CD40 expression per patient and for 3 out of 14 patients there was no detectable CD40 expression in the melanoma cells. Notably, CD40 expression in a patient's melanoma cells significantly and negatively correlates with the resistance level to ICB immunotherapy. To explore whether $R A S$ and $R A S$ - mediator expression levels correlate with $C D 40$ expression in a patient's melanoma cells, we further screened the mRNA expression profile of genes in the N-RAS regulation pathway microarray (Bio-Rad) (Fig. 7 f). Among the 20 genes examined, 4 of them (including NRAS, RAF1, HPRT1 and ITPR1) exhibited a significantly negative correlation with the CD40 expression level in melanoma cells, suggesting a potential negative regulatory role for RAS signaling in CD40 mRNA expression in melanoma cells from patient tumors. Together, these data suggest that melanoma cell-intrinsic CD40 correlates with response to ICB and response to inhibitors of the RAS-mediated pathways, including RGS, in melanoma patients.

\section{Discussion}

In this preclinical study, we first determined that RGS treatment reduces melanoma tumor growth and enhances response to ICB therapy. The combinatorial therapy of RGS + ICB exhibited stronger anti-tumor effects compared to ICB monotherapy and favored an inflammatory CD8 Tc cell response in the TME and TDLN (Fig. 4). We determined that the optimal in vivo dose of clinical-grade RGS [55] to treat melanoma tumors in mice by oral gavage is $300 \mathrm{mg} / \mathrm{kg} /$ day (dosed 5 days a week), which was well tolerated. This is consistent with the reported safety profile of RGS in clinical trials. Notably, in a recent phase II/III trial of metastatic pancreatic cancer [24] and phase III trial of MDS [21], patients received RGS $1800 \mathrm{mg} / \mathrm{m}^{2}$ via $2 \mathrm{~h}$ continuous IV infusions given twice weekly for 3 weeks of a four-week cycle or $1800 \mathrm{mg}$ per $24 \mathrm{~h}$ via $72 \mathrm{~h}$ continuous intravenous infusion administered every other week, which is equivalent to $\sim 600 \mathrm{mg} / \mathrm{kg}$ and $\sim 370 \mathrm{mg} / \mathrm{kg}$ in mouse, respectively [56]. An oral formulation of RGS (dose escalation to maximal dosing $560 \mathrm{mg}$ BID on days 1 to 14 of a 21-day cycle or 1 to 21 of a 28 -day cycle respectively) is being studied in phase I/Ila trials in head and neck squamous cell carcinoma (NCT01807546) and non-small cell lung cancer (NCT04263090). Given the promising safety and therapeutic profile of RGS combined with our data showing RGS can enhance response to ICB therapy in melanoma, our data indicate that a combination of RGS + ICB therapy warrants a clinical study in melanoma patients. Notably, tumor growth rate upon treatment with RGS alone was comparable to that of ICB, suggesting that treatment alone may be effective in slowing tumor growth for melanoma patients with primary or secondary resistance to ICB treatment.

While RGS reduced viability in vitro in human and murine melanoma cells independent of their mutational status (Fig. 1), we observed that RGS exhibited little inhibition of tumor growth in immunodeficient mice (Fig. 6 \& Suppl.Fig. 13). These data suggest not only that tumor cell-intrinsic effects of RGS are insufficient to restrain melanoma tumor growth in vivo, but also reinforce the key role of RGS-activation of anti-tumor immunity in its therapeutic role. Of note, dying cells are known to elicit a wound healing response in neighboring cells, so-called apoptosis-induced proliferation (AiP), that promotes proliferation of the surrounding cells [57]. Consistently, we observed a simultaneous increase of apoptotic (cleaved-caspase3) and mitotic (PLK1 and FOXM1) markers at $24 \mathrm{~h}$ post RGS treatment in melanoma cells (Fig. 1 c \&Suppl.Fig. 1). Based on the work in several model organisms, caspases drive AiP through 
ROS generation [58]. We observed RGS-induced caspases and ROS in melanoma cells. While a direct role of AiP has not been demonstrated here, AiP induction is a plausible mechanism for the RGS resistance we observed in the immunodeficient mice. Future work will need to address this question.

The work described here illustrates that RGS not only has direct melanoma cell killing effects, but it also induces immunogenic cell death to facilitate response to ICB immunotherapy, which is associated with elevated DC and Tc cell responses (Figs. 2, 3, 4). Notably, the RGS induced CD40 upregulation on melanoma cells, which is associated with cell death and tumor antigen release, indicating that $\mathrm{CD} 40$ was required for the induction of anti-tumor immune responses (Fig. 5). These data are consistent with the recent reports that CD40mediated signaling in tumor cells induces tumor cell death, while in normal cells, CD40 signaling is cytoprotective $[32,59]$. Of note, CD40 agonists have been combined with chemotherapy to enhance the capacity for $\mathrm{T}$ cell stimulation, especially in solid malignancies. However, no clinical benefit was observed with delivering chemotherapy prior to a CD40 agonist $[60,61]$. In contrast, preclinical models have shown that CD40 agonist treatment enhanced sensitivity to gemcitabine chemotherapy via the up-regulation of proinflammatory IFN $\gamma$ responses [61], which supports a role for delivery of CD40 agonists in sensitizing tumors for subsequent sequential therapies. Currently, several clinical trials are ongoing using the CD40 agonists APX005M: 1) in combination with nivolumab and cabiralizumab (CSF1R antagonist) in patients with advanced melanoma, nonsmall cell lung cancer or renal cell carcinoma whose disease has progressed on $\alpha-\mathrm{PD}-1 / \mathrm{PD}-\mathrm{L} 1$ therapy (NCT0302330); 2) in combination with a personal cancer vaccine (neo-pv-01) or ipilimumab with nivolumab in patients with advanced melanoma (NCT03597282); 3) in combination with nivolumab in patients with nonsmall cell lung cancer or metastatic melanoma (NCT03123783). It will be interesting to see how systemic activation of CD40 affects disease in these trials and to sort out mechanisms by which any observed effects occur.

Indeed, intratumoral $\mathrm{CD} 40$ activation in combination with ICB induces $\mathrm{T}$ cell-mediated eradication of melanoma in animal models [62]. However, safety concerns (e.g., thromboembolic events and cytokine storm release) limited the clinical development of some CD40 agonists early on [63]. Notably, when applying agonistic CD40 antibodies locally instead of systemically, adverse events were shown to be diminished [64, 65]. Our data show that CD40 expression is prognostic for therapeutic response to RGS, RAF inhibitor and ICB treatments in human melanoma cells (Fig. 7). The induction of CD40, as well as CD80 and ICOS-L, in RGS-treated melanoma tumors, may form a positive feedback loop to further sensitize tumors to RGS treatment. We identified a gain of CD40 copy number (CD40-gain) in 177 out of 358 TCGA-melanoma patients (Fig. 7 b). While we showed that inhibition of RAS-mediated pathways would induce CD40 on melanoma cells, based on prior work showing poor induction of CD40 expression in CD40 low cells $[27,28]$, induction of CD40 expression by RGS or other therapies may be more effective for those tumors that are CD40 diploid, or with increased CD40 copy number (CD40-gain). Recently, a systematic pan-tumor analyses investigated ICB-response biomarkers in a cohort of over 1000 ICB-treated patients from 12 individual studies, consisting of 7 tumor types, namely urothelial, head and neck, renal, colorectal, breast, non-small cell lung cancer and melanoma, treated with 3 distinct ICBclasses $(\alpha$-CTLA-4, PD-1, or PD-L1) [66]. This metaanalysis study revealed the importance of both tumorcell-intrinsic (e.g., tumor mutational burden and PD-L1 expression) and tumor-cell-extrinsic (e.g., CXCL9, CD8A, CXCL13, CCR5 and T cell inflamed gene expression signature) factors underpinning ICB sensitization. While ICB may reinvigorate $\mathrm{T}$ cells by releasing the brake on $\mathrm{T}$ cell activation, many of these ICB-response factors may further modulate the response and the final therapeutic outcome. In our preclinical melanoma models, both B16F10 and YUMM3.3 tumors do not respond to ICB monotherapy (Fig. 4). The targeted therapy of rigosertib increased response to ICB and converts the "non-responders" into "responders".

There are limitations to our study. For instance, the analysis of protein markers and immune profiling in melanoma tumors should be interpreted with caution; at the end point of tumor tissue collection, some of the tumors were large with regions of necrosis (particularly in the control group). This and other potential variables that are not directly related to therapy may influence the microenvironment within the tumors and increase variability among tumors. Another limitation is that clinical trials to test the therapeutic effects of RGS in melanoma have not occurred at this time. Due to the small sample size of paired BRAF inhibitor samples from the BRAF inhibitor clinical trial, we could only identify a trend toward a correlation between $\mathrm{CD} 40^{+}$melanoma and patient survival, which warrants future trials to validate the role of $\mathrm{CD}_{4} 0^{+}$melanoma cells in treatment responses. Moreover, there is recent controversy around the microtubule-destabilizing activity of RGS preparations as to whether it is due to a contaminant or endogenous activity of RGS $[67,68]$. Of note, several clinical trials are investigating the effectiveness of combining microtubule inhibitors with ICB therapy in melanoma (NCT01827111, NCT00796991 and NCT02617849). 
Here we are predominately studying the effect of RGS on ICD and downstream immune responses, so regardless of whether RGS is inducing ICD by directly inhibiting the RAS/RAF/MAPK/PI3K pathway or microtubules, the results are highly significant.

One particularly interesting finding from our study is the unexpected upregulation of CD40 in the melanoma tumor cells following inactivation of RAS-mediated signaling by RGS or dabrafenib plus trametinib treatment. Notably, RGS does not inhibit kinase activities of A-Raf, B-Raf, or C-Raf when added in vitro to purified Raf proteins $[18,38]$. However, the level of Raf dimerization was dramatically reduced in HeLa cells with EGF stimulation [38]. It is well established that the RAS/RAF/MEK pathway is essential for the proliferation, survival, and progression of most tumor types. We postulated initially that suppression of the RAS/RAF/MEK and PI3K pathways might promote immune surveillance through increased antigen release from dying tumor cells. However, we clearly cannot rule out the possibility that CD40 upregulation itself is the key event associated with the RGS-initiated suppression of tumor growth. In fact, under otherwise identical conditions, response to RGS was reversed by (i) suppression of CD40 expression in the tumor cells by shRNA, and (ii) depletion of $\mathrm{CD}^{+} \mathrm{Tc}$ cells. Both results are consistent with the hypothesis that RGS markedly enhanced immune surveillance of the tumor through CD40-upregulation. Given the importance of RAS/RAF/PI3K pathway activation across a very broad spectrum of human cancer types, it is worth considering the possibility that, in addition to its effects on cell proliferation/survival, RAS/RAF/PI3K-pathway activity also directly masks immune surveillance during the early stages of tumor progression.

\section{Conclusions}

This study provides proof of concept that the inhibition of RAS/RAF/PI3K-pathway induces CD40 upregulation and immunogenic cell death in melanoma cells, which sensitizes them to ICB therapy. The observations here point to the potential usefulness in examining CD40 expression as a possible prognostic marker for response to ICB therapy as well as a need for new clinical trials combining RGS with ICB in metastatic melanoma patients.

\section{Materials and methods Study design}

The aim of the study was to determine the effectiveness and mechanism of RGS as a monotherapy and in combination with ICB in melanoma. Cell viability and cell death assays through flow cytometry, western blotting and IHC staining supported the cytotoxic effect of RGS on melanoma cells, but not the normal melanocytes. Detailed immune profiling through RNA-Seq, flow cytometry and IHC staining of tumor and lymph node samples was used to determine the role of RGS in shaping the TME and the results were confirmed with CD8 cell depletion. Cytokine array, cell lineage tracking by flow cytometry and shRNA knockdown were used to identify the requirement of $\mathrm{CD}_{4} 0^{+}$melanoma cells in RGS-induced immune responses. Patient-derived xenograft models and multiplex IHC analysis of melanoma tumor samples from BRAF inhibitor trial were used to validate the role of $\mathrm{CD} \mathrm{O}^{+}$melanoma in human. Lastly, we conducted human melanoma database analysis, including TCGA, Oncomine and Single Cell RNA-Seq Portal, to determine the prognostic value of melanomaexpressing CD40 in patient survival and response to RAF inhibition and ICB therapies.

\section{Cell lines}

Human melanoma cell lines [A375; SkMel2; SkMel5; HS294T], the B16F10 murine melanoma cell line and the HEK293 cell line were purchased from the American Tissue Culture Collection (ATCC). Murine melanoma cell lines [YUMM 2.1; YUMM 3.3; YUMM 4.1; YUMM 5.2] were provided by Marcus Bosenberg (Yale University). The status of driver mutation genes in melanoma cell lines were listed in Suppl. Table 1. The genetics of the YUMM cell lines was verified by RNA-Seq. Human Mel-ST and murine Melan-A melanocytes were provided by Robert Weinberg (Massachusetts Institute of Technology) and Dorothea Bennett (St. George's Hospital Medical School), respectively. All cell lines were free of mycoplasma contamination.

\section{Mouse tumor models}

Animal studies were approved by the Vanderbilt Institutional Care and Animal Use Committee (IACUC) and were performed in accordance with Vanderbilt IACUC guidelines. All animals were housed under pathogen-free conditions at the Vanderbilt Animal Care Facility. C57BL/6 mice, athymic nude mice, CD40 knockout, and NSG mice were purchased from Jackson Labs. Tumor xenografts were established in 8-10 week-old male or female mice. For the in vivo melanoma model, mice received $3 \times 10^{5}$ of tumor cells in $100 \mu \mathrm{l}$ of serum-free DMEM medium by subcutaneous injection in the lower back. Mouse body weight was assessed once a week, and tumor measurements were taken twice a week with micro-calipers. Tumor volume was estimated as 0.5 *length"width"width. Treatment began when tumors reached $\sim 100 \mathrm{~mm}^{3}$ volume on average or at day 10 post tumor cell inoculation and continued until tumors in the experiment exceeded $15 \mathrm{~mm}$ in diameter or became perforated. 


\section{Patient material}

All patient donors signed an approved informed consent before providing tissue samples. Patient samples were collected on a tissue-collection protocol approved by the Vanderbilt University IRB. Melanoma PDXs in NSG mice were established from melanoma tumors obtained from patients who were referred for surgical resection or biopsy and propagated, as described previously [51, 69]. The status of driver mutation genes in melanoma PDXs were listed in Suppl. Table 1. Paired advanced melanoma samples from 11 patients (pre- and post-treatment of BRAF inhibitors) were collected as part of NCT01205 815 clinical trial (Suppl.Table 2).

\section{Therapeutic treatment regimens}

Rigosertib (300 mg/kg, Onconova Therapeutics) was administered 5 days a week by oral gavage in a total volume of $100 \mu \mathrm{L}$. Immunotherapies or equivalent amounts of isotype control, including anti-mouse PD-1 (clone: RMP1-14), Rat IgG2a control (clone: 2A3), anti-mouse CTLA-4 (clone: 9H10) and polyclonal Syrian hamster IgG control, were administered intraperitoneally at $100 \mu \mathrm{g}$ per mouse every 3 days for two weeks. For depletion of $\mathrm{CD}^{+}$and $\mathrm{CD}^{+}$cells, mice were injected intraperitoneally with $200 \mu \mathrm{g}$ of anti-mouse CD4 (clone: GK1.5), anti-mouse CD8 $\alpha$ (clone: YTS 169.4), or the same amount of rat IgG2b control (clone: LTF-2) antibodies 3 days prior to the tumor cell injection and every 3 days thereafter for the entire experiment. All antibodies were purchased from BioXcell. For caspase inhibition, pan-caspase inhibitor Z-VAD (APExBIO Technology), or the same amount of DMSO control in PBS, were given intra-peritoneally daily at $2 \mathrm{mg} / \mathrm{kg}$ for 14 consecutive days. PP242 (SelleckChem, \#S2218), Everolimus (SelleckChem, \#S1120), Dabrafenib (NCI), Trametinib (NCI) and Ipatasertib (MedKoo, \#205467) were reconstituted in dimethyl sulfoxide (DMSO) and used for in vitro culture.

\section{Western blot}

RIPA buffer (Sigma-Aldrich) was used to prepare lysates and protein concentration was measured using Bradford Protein Assay reagent (BioRad). Whole-cell extracts were separated on 4-20\% Mini-Protean TGX Gels (BioRad) and transferred using Trans-Blot Turbo RTA Transfer Kit (Nitrocellulose, BioRad). Primary antibodies were used at 1:500 dilution, except antibodies recognizing housekeeping protein HSP90, which were used at 1: 2000. All primary antibodies were applied overnight at $4{ }^{\circ} \mathrm{C}$. See Suppl. Table 3 for primary antibodies utilized in this study. Secondary anti-rabbit or anti-mouse antibodies were used at 1:5000 dilutions for $1-2 \mathrm{~h}$ at room temperature.

\section{Cell viability assays}

The CellTiter-Blue ${ }^{\bullet}$ Cell Viability Assay was performed according to the manufacturer's recommendations. The percentages of viable cells and IC50s were calculated and presented in relevance to untreated control (100\% viable) using Prism 8.0.1 software (Graphpad). Crystal violet staining was performed with a commercial $1 \%$ solution (Sigma-Aldrich) on cells that were fixed with $100 \%$ methanol for $10 \mathrm{~min}$. Images were captured using a EVOS XL Core Imaging System (Invitrogen).

\section{Flow cytometric analysis}

Tumor tissues were minced on a programmable gentleMACS dissociator (Miltenyi Biotec) and digested with an enzyme solution of collagenase I (1,500 CDU, Worthington Biochemical Corp.), Dispase II $(1 \mathrm{mg} / \mathrm{mL}$, Worthington Biochemical Corp.) and DNase I (0.1 mg/ mL, Worthington Biochemical Corp.). Digested tumors were passed through a $70-\mu \mathrm{m}$ strainer to obtain a singlecell suspension. Minced samples of mouse spleens and lymphoid nodes (LN) were pressed through a 70- $\mu \mathrm{m}$ strainer using a syringe plunger to obtain a single-cell suspension. Bone marrow (BM) was flushed with 5\% FBS RPMI, centrifuged, resuspended, and passed through a $70-\mu \mathrm{m}$ strainer for single-cell suspension. Erythrocytes were lysed by incubation in an ammoniumchloride-potassium buffer for $5 \mathrm{~min}$ at room temperature. The detail of antibodies used (Suppl.Table 4), staining, and flow cytometry analyses protocols is according to our previously published methodology [70]. Briefly, cells were incubated with Ghost Dye Violet 510 (Tonbo Biosciences), an amine-reactive viability dye used to discriminate live/dead cells and washed with FACS buffer (PBS containing 1\% v/v FBS). After blocking Fc receptors with anti-mouse CD16/CD32 mAb (BD Biosciences) in FACS buffer for $20 \mathrm{~min}$, cells were incubated with target antibodies. After staining, cells were washed twice in FACS buffer and fixed with $300 \mu \mathrm{l} /$ tube of fixation buffer (1\% formalin in PBS). Data were collected using a BD LSR Fortessa flow cytometer and analyzed using FlowJo software (Version 10.5.3). For t-SNE analysis, live $\mathrm{CD}_{4} 5^{+}$leukocytes were concatenated after downsampling to $12,500 \sim 20,000$ events for subsequent high-dimensional data analysis to normalize the contribution among samples under different treatments. Samples were then analyzed in parallel by t-SNE and manually gated leukocyte populations were overlaid onto the total t-SNE map using FlowJo 10.5.3. For cell culture samples, apoptosis was assessed using an Annexin VFITC Apoptosis Detection Kit (eBioscience). Reactive oxygen species (ROS) production was determined using the MitoSOX ${ }^{\mathrm{Tm}}$ Red reagent (Invitrogen). For intracellular flow cytometric analysis on the cytokine production and antigen specificity of tumor-infiltrating $\mathrm{CD}^{+}$Tc cells, 
we followed the protocol as described previously [71]. Frozen cells isolated from YUMM3.3 and B16F10 tumors ( $n=3$ per treatment group), containing 0.2 million $\mathrm{T}$ cells, were thawed and recovered in complete RPMI medium with $10^{2} \mathrm{U} / \mathrm{ml}$ mouse recombinant IL-2 (Biolegend, \#714604) overnight. These cells were then cocultured with or without parental tumor irradiated cells (50 Gy) for stimulation in 5:1 ratio of T-cell: tumor-cell in irradiated medium. Protein transport inhibitors, including $1 \mathrm{ul} / \mathrm{ml}$ Monesin (BD BIOSCIENCES, \# 554724) and $1 \mathrm{ul} / \mathrm{ml}$ Brefeldin A (BD BIOSCIENCES, \# 555029) were added into the co-cultures. Cells were incubated for $4 \mathrm{~h}$ at $37^{\circ} \mathrm{C}$ and transferred to $4{ }^{\circ} \mathrm{C}$ overnight. PBMCs isolated from tumor-free C57BL/6 mice were used as a negative control for antigen specificity. The stimulation with $\alpha \mathrm{CD} 3 / \mathrm{CD} 28$ beads (Gibco, \# 11456D, 1:1 bead-to-cell ratio) was used as a positive control for $\mathrm{CD}^{+}{ }^{+} \mathrm{Tc}$ cell responses. The levels of IFN- $\gamma$, TNF- $\alpha$, and granzyme B were assessed using a BD LSR Fortessa flow cytometer and analyzed using FlowJo software (Version 10.5.3).

\section{RNA sequencing}

Tumors were collected into RNAlater. RNA was extracted using Qiagen RNAeasy plus mini Kit for three replicates from $\mathrm{H}_{2} \mathrm{O}$ and Rigosertib treated groups. Samples were submitted to Vanderbilt Technologies for Advanced Genomics (VANTAGE) for RNA QC, library prep and sequencing with a mean of $\sim 30 \mathrm{M}$ reads/sample. Samples were sequenced on the Illumina NovaSeq 6000 using $150 \mathrm{bp}$ paired-end reads. Reads were then aligned to the STAR aligner for reads. FASTQ files containing raw reads from the RNA-seq analyses have been deposited with the NCBI GEO under accession GSE149737. Gene set enrichment analyses from the RNA-seq data were performed according to the instructions (https://geneontology.org/).

\section{Pathology and immunohistochemistry assessment}

AST and ALT analysis, complete blood cell count and immunohistochemistry (IHC) analysis on 10\% buffered formalin-fixed, paraffin-embedded tissue sections were performed by the Vanderbilt Translational Pathology Shared Resource [51]. Slides were placed on the Leica Bond Max IHC stainer. All steps besides dehydration, clearing and coverslipping are performed on the Bond Max. Slides are deparaffinized. Heat induced antigen retrieval was performed on the Bond Max using their Epitope Retrieval 2 solution for $20 \mathrm{~min}$. Slides were incubated with primary antibody (Suppl.Table 3). The Bond Polymer Refine detection system (DS9800, Leica, Buffalo Grove, IL) was used for visualization. Slides were the dehydrated, cleared and coverslipped. IHC slides were scanned at the Vanderbilt Digital Pathology Shared
Resource. The automated quantification of the percentages of the target-positive cells was performed by Leica Biosystems' Digital Image Hub at Vanderbilt, using the software available with the Leica SCN400 Slide Scanner. For multiplex IHC, sequential cycles of IHC staining of antibodies were conducted. The Bond Polymer Refine detection system and the Vector AEC (3-amino-9-ethylcarbazole) HRP Substrate (SK-2405, Vector Lab, Burlingame, CA) was used for visualization. After each cycle of staining, the full slides were immediately scanned and antibodies were stripped from the section, eliminating the risk of antibody cross-reactivity when performing the next round of labelling. Hematoxylin was used in every cycle of staining for cell identification and matching. Computational cell segmentation and images processing were performed by Leica Biosystems' Digital Image Hub at Vanderbilt. Fiji/ImageJ (version 1.52p) was used for image pseudocoloring and overlay.

\section{Cytokine array}

Tumor lysates, serum, and cell culture supernatant samples were examined in cytokine arrays using Mouse Cytokine Array G3 Kit (62 cytokines) (RayBiotech), per the manufacture's protocol. For each mouse tissue, samples were pooled from 5 mice per group.

\section{Cells with stable CD40 knockdown}

For specific knockdown of CD40, plasmids (Sigma-Aldrich) of retroviral vectors carrying CD40 shRNA against coding sequence (CDS), untranslated region (UTR) or random sequence as control shRNA were developed to infect HEK293T cells with FugeneHD (Promega). Viruscontaining supernatants were used to transduce YUMM3.3 melanoma cells twice, $24 \mathrm{~h}$ apart with $6 \mu \mathrm{g} /$ $\mathrm{ml}$ polybrene. Twenty-four hours after the final transduction, stable cells expressing the shRNA were isolated via selection in the presence of $1 \mu \mathrm{g} / \mathrm{ml}$ puromycin (RPI Corp). The expression of CD40 protein in the stable cells was evaluated at mRNA and cell surface protein levels. The cells that showed $\geq 60 \%$ reduction of CD 40 were used for experiments.

\section{Publicly available database analysis}

Whole-genome copy number alteration (CNA) analysis, microarray, and RNA sequencing-based gene expression and mutation burden profiling in melanoma (TCGA) were obtained from cBioPortal for Cancer Genomics (www.cbioportal.org). Kaplan-Meier survival curves were used to show the effect of target genes on the outcomes of melanoma patients. For drug sensitivity correlation analysis, human cancer cell line datasets published in Oncomine (www.oncomine.org) was used. For RNASeq dataset analysis, two bulk RNA-seq datasets, which studied resistance mechanisms of immune checkpoint 
blockade [52, 53], were downloaded from the original literature and collected into the Immu-Mela database (https://bioinfo.vanderbilt.edu/database/Immu-Mela/).

The association of CD40 expression and immunotherapy response in these two datasets were evaluated and obtained from the Immu-Mela database. The scRNA-seq data and the overall expression of immune resistance score (OE.immune_resistance) were downloaded from Single Cell Portal (https://singlecell.broadinstitute.org/ single_cell/study/SCP109/melanoma-immunotherapyresistance\#study-summary) [54]. As described in the original paper [54], gene expression was quantified as $\log _{2}(\mathrm{TPM} / 10+1)$, where transcripts per million (TPM) was divided by 10 and $\log 2$-transformed after the addition of 1 . Overall expression of immune resistance (OE.immune_resistance) score was computed for the variation in the signal-to-noise ratio across hallmark genes of immune evasion, suppression, and exclusion in malignant cells [54]. Spearman correlation was used to calculate the association between CD40 expression and the OE.immune_resistance score.

\section{Statistical analysis}

Treatment effects in standard two-group experiments were compared using a two-sample t-test between independent samples or a paired t-test between correlated samples. A one-way analysis of variance (ANOVA) with post hoc Tukey's HSD test was used for more than twogroup experiments to compare treatments' differences. For in vivo experiments, the progression of tumor volume $\left(\mathrm{mm}^{3}\right)$ over time among groups of mice receiving different therapies were compared with a linear mixedeffects regression model to account for within mouse correlation. A square root or a natural log transformation was implemented to better meet the normality assumptions. The likelihood ratio test was performed to identify statistically significant time by treatment effects. A statistically significant interaction implies that the magnitude of treatment differences depends on the actual day of measurement. Using model-based (leastsquare) means, average tumor growth between treatment groups was estimated and compared with the Wald test. For the synergy test, a mixed-effect model with individual drug effects and the interactive effect over time was fitted to evaluate the interaction between two drugs in tumor growth rate where tumor volume was analyzed on a natural log scale. The adjusted average tumor growth rates were estimated for individual drugs and the combination drug based on the model. The likelihood ratio test was performed to determine if drug interactions were statistically significant $(p<0.05)$. A statistically significant interaction implies the effect of drug combinations is supra-additive (synergistic) when the interaction plot (Fig. 4 e) showed lines are in the same direction, but one is steeper than the other. The Bonferroni correction was used to control the experiment-wise type I error rate at $5 \%$. Residual analysis was performed to evaluate model assumptions. Survival curves are estimated using the Kaplan-Meier method and compared statistically using the log-rank test. For RNA-Seq experiments, pre-filtering of the dataset was conducted by only including genes with a median count of 5 or more in at least one group to increase the speed of the transformation and testing functions within DESeq2 [72]. Raw count data were used in DESeq2, implementing the negative binomial generalized linear model to test for differential gene expression. The Benjamini-Hochberg (BH) approach was used to adjust the $p$-value [73]. Spearman's rank correlation was used to measure the strength and direction of a monotonic association between two ranked variables. All tests of statistical significance were two-sided. GraphPad's Prism 8.0.1 software and $\mathrm{R}$ language (version 3.3.0.) were used for the statistical analysis. Data are presented with mean with SE. * $p \leq 0.05 ;{ }^{* * *} p \leq 0.01 ;{ }^{* * * * *} p \leq 0.001 ;{ }^{* * * * * *} p \leq 0.0001$.

\begin{abstract}
Abbreviations
Ag: Antigen; AiP: Apoptosis-induced proliferation; ALT: Alanine aminotransferase; APCs: Antigen-presenting cells; AST: Aspartate aminotransferase; BRAF: V-raf murine sarcoma viral oncogene homolog B1; Bregs: Regulatory B cells; CD: Cluster of differentiation; CD40L: CD40 ligand; CDC: Conventional dendritic cells; CDS: Coding sequence; CTLA-4: Cytotoxic T-lymphocyte-associated protein 4; DC: Dendritic cells; ER: Endoplasmic reticulum; FOB: Follicular B cell; GzmB: Granzyme B; HDAC: Histone deacetylase; ICB: Immune checkpoint blockade; ICD: Immunogenic cell death; ICOS: Inducible T-cell costimulator; IFN: Interferon; IL: Interleukin; MDS: Myelodysplastic syndrome; MHC: Major histocompatibility complex; NRAS: Neuroblastoma RAS viral oncogene homolog; OS: Overall survival; PD1: Programmed cell death protein 1; pDC: Plasmacytoid dendritic cells; PDL1: Programmed cell death protein 1 ligand 1; PDX: Patient-derived xenografts; PFS: Progression free survival; PI3K: Phosphoinositide 3-kinase; PLK1: Polo-like kinase 1; RBD: RAS-binding domain; RGS: Rigosertib; ROS: Reactive oxygen species; sc: Single cell; Tc: Cytotoxic T; TCGA: The cancer genome atlas; TDLN: Tumor-draining lymph node; Th: T helper; TME: Tumor microenvironment; TNF: Tumor necrosis factor; Tregs: Regulatory T cells; UTR: $3^{\prime}$ untranslated region
\end{abstract}

\section{Supplementary Information}

The online version contains supplementary material available at https://doi org/10.1186/s12943-021-01366-y.

Additional file 1: Supplementary Figure 1. Rigosertib promotes cel death, ROS production and inhibits AKT activation as well as mitosis of melanoma cells in vitro.

Additional file 2: Supplementary Figure. 2. Rigosertib demonstrates a general anti-tumor effect in mice.

Additional file 3: Supplementary Figure. 3. RGS promotes plasminogen activating cascade and synaptic vesicle trafficking in the TME.

Additional file 4: Supplementary Figure 4. Rigosertib promotes conventional dendritic cells and T cells in the tumor-draining lymph nodes.

Additional file 5: Supplementary Figure. 5. Rigosertib-induced caspase-dependent cell death is important for the anti-tumor responses in vivo. 
Additional file 6: Supplementary Figure. 6. Rigosertib demonstrates a safe profile in healthy and tumor-bearing mice.

Additional file 7: Supplementary Figure. 7. Rigosertib plus ICB treatment induces NK and T cell responses in the tumor draining lymph nodes (TDLN)

Additional file 8: Supplementary Figure. 8. No Toxicity of Rigosertib + ICB combinational treatment in vivo.

Additional file 9: Supplementary Figure. 9. Cytokine production and antigen (Ag) specificity of tumor-infiltrating $\mathrm{CD}^{+}$cytotoxic T cells (Tc).

Additional file 10: Supplementary Figure. 10. Rigosertib $+I C B$ combinatorial treatment reduces PD- $\mathrm{L} 1^{+}$Bregs.

Additional file 11: Supplementary Figure. 11. Characterization of CD40 knockdown clones.

Additional file 12: Supplementary Figure. 12. CD40 ligand (CD4OL) expression in tumor-infiltrating $T$ cells.

Additional file 13: Supplementary Figure. 13. Intact adaptive immune system is required for rigosertib to inhibit melanoma tumor growth in vivo.

Additional file 14: Supplementary Figure. 14. Copy number of CD40 prognoses sensitivity of human melanoma cells to the RAF inhibitors.

Additional file 15: Supplementary Figure. 15. CD80 and ICOSL do not prognose sensitivity of human melanoma cells to the RAF inhibitors.

Additional file 16: Supplementary Figure. 16. Bulk tumor RNA-Seq analysis of two datasets with both transcriptomics and immunotherapy response in melanoma

Additional file 17: Supplementary Table 1. Genetic background of melanoma models used in this study

Additional file 18: Supplementary Table 2. Patient characteristics and response to targeted therapy

Additional file 19: Supplementary Table 3. List of antibodies used in western blotting and $\mathrm{IHC}$ staining

Additional file 20: Supplementary Table 4. List of antibodies used in flow cytometry

\section{Acknowledgments}

We thank Dr. Albert B. Reynolds for his helpful discussions and editing of the manuscript. We thank Dr. Jin Chen for providing reagents, as well as Drs. Qi Liu and Jing Yang at VANGARD (Vanderbilt Technologies for Advanced Genomics Analysis and Research Design) for providing support on RNA-Seq analysis. The authors sincerely thank Onconova Therapeutics, Newtown, PA 18940, for kindly supplying rigosertib for this work. We also acknowledge the Translational Pathology Shared Resource (TPSR) and the Immune Phenotyping core supported by NCl/NIH Cancer Center Support Grant 5P30 CA6848519 and the Vanderbilt Mouse Metabolic Phenotyping Center Grant 2 U24 DK059637-16. The Shared Instrumentation Grant S10 OD023475-01A1 for the Leica Bond RX. Whole slide imaging and quantification of immunostaining were performed in the Digital Histology Shared Resource at Vanderbilt University Medical Center (www.mc.vanderbilt.edu/dhsr).

\section{Authors' contributions}

CY and AR designed the research approach; CY, NS and JY performed the experiments; CY, CAN and JTR analyzed data; SC, GDA, and CY performed statistical analyses; DBJ, EPR, JTR and RLS provided key resources; CY made a draft of the paper; CY, AR, RLS, SC, GDA and AEV revised and edited the paper. The authors read and approved the final manuscript.

\section{Funding}

This work was supported by a VA Merit award (I01BX002301) and SRCS award to AR and by CA116021 (AR), and by R37CA233770 (AEV).

\section{Availability of data and materials}

All data generated during this study are included in this published article and its supplementary files. FASTQ files containing raw reads from the RNAseq analyses have been deposited with the NCBI GEO under accession GSE149737.

\section{Declarations}

Ethics approval and consent to participate

Animal studies were approved by the Vanderbilt Institutional Care and Animal Use Committee (IACUC) and were performed in accordance with Vanderbilt IACUC guidelines. All patient donors signed an approved informed consent before providing tissue samples. Patient samples were collected on a tissue-collection protocol approved by the Vanderbilt University IRB.

\section{Consent for publication}

Not applicable.

\section{Competing interests}

EPR is a member of the Board of Directors of Onconova, receives grant funding from Onconova, and is a paid consultant for Onconova. All other coauthors have declared that no additional conflict of interest exists.

\section{Author details}

${ }^{1}$ Department of Veterans Affairs, Tennessee Valley Healthcare System, 432 PRB, 2220 Pierce Ave, Nashville, TN 37232, USA. Department of Pharmacology, Vanderbilt University School of Medicine, Nashville, TN, USA. ${ }^{3}$ Department of Medicine, Division of Hematology and Oncology, Vanderbilt University Medical Center, Nashville, TN, USA. ${ }^{4}$ Department of Pathology, The Ohio State University, Columbus, OH, USA. ${ }^{5}$ Department of Oncological Sciences, Icahn School of Medicine at Mount Sinai, New York, NY, USA. ${ }^{6}$ Departments of Surgery and Pediatrics and the Epithelial Biology Center, Vanderbilt University School of Medicine, Nashville, TN, USA. ${ }^{7}$ Department of Biostatistics, Vanderbilt University Medical Center, Nashville, TN, USA.

Received: 15 January 2021 Accepted: 19 April 2021

Published online: 06 June 2021

\section{References}

1. Reddy BY, Miller DM, Tsao H. Somatic driver mutations in melanoma. Cancer. 2017;123(S11):2104-17. https://doi.org/10.1002/cncr.30593.

2. Millis SZ, Ikeda S, Reddy S, Gatalica Z, Kurzrock R. Landscape of phosphatidylinositol-3-kinase pathway alterations across 19784 diverse solid tumors. JAMA Oncol. 2016;2(12):1565-73. https://doi.org/10.1001/jama oncol.2016.0891.

3. Long GV, Hauschild A, Santinami M, Atkinson V, Mandala M, Chiarion-Sileni $\checkmark$, et al. Adjuvant dabrafenib plus trametinib in stage III BRAF-mutated melanoma. N Engl J Med. 2017;377(19):1813-23. https://doi.org/10.1056/ NEJMoa1708539.

4. Larkin J, Ascierto PA, Dreno B, Atkinson V, Liszkay G, Maio M, et al. Combined vemurafenib and cobimetinib in BRAF-mutated melanoma. $\mathrm{N}$ Engl J Med. 2014;371(20):1867-76. https://doi.org/10.1056/NEJMoa1408868.

5. Charles J, Martel C, de Fraipont F, Leccia MT, Robert C, Busser B. Mechanisms of resistance to anti-BRAF treatments. Ann Dermatol Venereol. 2014;141(11):671-81. https://doi.org/10.1016/j.annder.2014.06.021.

6. Hernandez-Davies JE, Tran TQ, Reid MA, Rosales KR, Lowman XH, Pan M, et al. Vemurafenib resistance reprograms melanoma cells towards glutamine dependence. J Transl Med. 2015;13(1):210. https://doi.org/10.11 86/s12967-015-0581-2.

7. Manzano JL, Layos L, Buges C, de Los Llanos Gil M, Vila L, Martinez-Balibrea $\mathrm{E}$, et al. Resistant mechanisms to BRAF inhibitors in melanoma. Ann Transl Med. 2016:4:237

8. Wolchok J. How recent advances in immunotherapy are changing the standard of care for patients with metastatic melanoma. Ann Oncol. 2012; 23(Suppl 8):viii15-21.

9. Hodi FS, O'Day SJ, McDermott DF, Weber RW, Sosman JA, Haanen JB, et al. Improved survival with ipilimumab in patients with metastatic melanoma. N Engl J Med. 2010;363(8):711-23. https://doi.org/10.1056/NEJMoa1003466.

10. Larkin J, Chiarion-Sileni V, Gonzalez R, Grob JJ, Rutkowski P, Lao CD, et al. Five-year survival with combined nivolumab and ipilimumab in advanced melanoma. N Engl J Med. 2019;381(16):1535-46. https://doi.org/10.1056/ NEJMoa1910836.

11. Marin-Acevedo JA, Chirila RM, Dronca RS. Immune checkpoint inhibitor toxicities. Mayo Clin Proc. 2019;94(7):1321-9. https://doi.org/10.1016/j.ma yocp.2019.03.012. 
12. Dummer R, Schadendorf D, Ascierto PA, Arance A, Dutriaux C, Di Giacomo AM, et al. Binimetinib versus dacarbazine in patients with advanced NRASmutant melanoma (NEMO): a multicentre, open-label, randomised, phase 3 trial. Lancet Oncol. 2017;18(4):435-45. https://doi.org/10.1016/S1470-2045(1 7)30180-8.

13. Greger JG, Eastman SD, Zhang V, Bleam MR, Hughes AM, Smitheman KN, et al. Combinations of BRAF, MEK, and PI3K/mTOR inhibitors overcome acquired resistance to the BRAF inhibitor GSK2118436 dabrafenib, mediated by NRAS or MEK mutations. Mol Cancer Ther. 2012;11(4):909-20. https://doi. org/10.1158/1535-7163.MCT-11-0989.

14. Sosman JAKM, Lolkema MPJ, et al. A phase $1 \mathrm{~b} / 2$ study of LEE011 in combination with binimetinib (MEK162) in patients with NRAS-mutant melanoma: early encouraging clinical activity. J Clin Oncol. 2014;32(15_ suppl):9009. https://doi.org/10.1200/jco.2014.32.15_suppl.9009.

15. Xu F, He Q, Li X, Chang CK, Wu LY, Zhang Z, et al. Rigosertib as a selective anti-tumor agent can ameliorate multiple dysregulated signaling transduction pathways in high-grade myelodysplastic syndrome. Sci Rep. 2014;4:7310

16. Hyoda T, Tsujioka T, Nakahara T, Suemori S, Okamoto S, Kataoka M, et al. Rigosertib induces cell death of a myelodysplastic syndrome-derived cell line by DNA damage-induced G2/M arrest. Cancer Sci. 2015;106(3):287-93. https://doi.org/10.1111/cas.12605.

17. Chapman CM, Sun X, Roschewski M, Aue G, Farooqui M, Stennett L, et al. ON 01910.Na is selectively cytotoxic for chronic lymphocytic leukemia cells through a dual mechanism of action involving PI3K/AKT inhibition and induction of oxidative stress. Clin Cancer Res. 2012;18(7):1979-91. https:// doi.org/10.1158/1078-0432.CCR-11-2113.

18. Athuluri-Divakar SK, Vasquez-Del Carpio R, Dutta K, Baker SJ, Cosenza SC, Basu I, et al. A small molecule RAS-mimetic disrupts RAS association with effector proteins to block signaling. Cell. 2016;165(3):643-55. https://doi. org/10.1016/j.cell.2016.03.045.

19. Prasad A, Khudaynazar N, Tantravahi RV, Gillum AM, Hoffman BS. ON 01910. $\mathrm{Na}$ (rigosertib) inhibits PI3K/Akt pathway and activates oxidative stress signals in head and neck cancer cell lines. Oncotarget. 2016;7(48):79388400. https://doi.org/10.18632/oncotarget.12692.

20. Dai YH, Hung LY, Chen RY, Lai CH, Chang KC. ON 01910.Na inhibits growth of diffuse large B-cell lymphoma by cytoplasmic sequestration of sumoylated C-MYB/TRAF6 complex. Transl Res. 2016;175:129-43 e113. https://doi.org/10.1016/j.trsl.2016.04.001.

21. Garcia-Manero G, Fenaux P, Al-Kali A, Baer MR, Sekeres MA, Roboz GJ, et al. Rigosertib versus best supportive care for patients with high-risk myelodysplastic syndromes after failure of hypomethylating drugs (ONTIME): a randomised, controlled, phase 3 trial. Lancet Oncol. 2016;17(4): 496-508. https://doi.org/10.1016/S1470-2045(16)00009-7.

22. Silverman LR, Greenberg P, Raza A, Olnes MJ, Holland JF, Reddy P, et al. Clinica activity and safety of the dual pathway inhibitor rigosertib for higher risk myelodysplastic syndromes following DNA methyltransferase inhibitor therapy. Hematol Oncol. 2015;33(2):57-66. https:/doi.org/10.1002/hon.2137.

23. Jimeno A, Li J, Messersmith WA, Laheru D, Rudek MA, Maniar M, et al. Phase I study of ON 01910.Na, a novel modulator of the polo-like kinase 1 pathway, in adult patients with solid tumors. J Clin Oncol. 2008;26(34):550410. https://doi.org/10.1200/JCO.2008.17.9788.

24. O'Neil BH, Scott AJ, Ma WW, Cohen SJ, Leichman L, Aisner DL, et al. A phase $\|/ I\|$ randomized study to compare the efficacy and safety of rigosertib plus gemcitabine versus gemcitabine alone in patients with previously untreated metastatic pancreatic cancer. Ann Oncol. 2015;26(12):2505. https://doi.org/1 0.1093/annonc/mdv477.

25. Ohnuma T, Lehrer D, Ren C, Cho SY, Maniar M, Silverman L, et al. Phase 1 study of intravenous rigosertib (ON 01910.Na), a novel benzyl styryl sulfone structure producing G2/M arrest and apoptosis, in adult patients with advanced cancer. Am J Cancer Res. 2013;3(3):323-38.

26. Tong AW, Stone MJ. Prospects for CD40-directed experimental therapy of human cancer. Cancer Gene Ther. 2003;10(1):1-13. https://doi.org/10.1038/sj. cgt.7700527.

27. Kalbasi A, Fonsatti E, Natali PG, Altomonte M, Bertocci E, Cutaia O, et al. CD40 expression by human melanocytic lesions and melanoma cell lines and direct CD40 targeting with the therapeutic anti-CD40 antibody CP870,893. J Immunother. 2010;33(8):810-6. https://doi.org/10.1097/CJ.0b013 e3181ee73a7.

28. von Leoprechting A, van der Bruggen P, Pahl HL, Aruffo A, Simon JC. Stimulation of CD40 on immunogenic human malignant melanomas augments their cytotoxic T lymphocyte-mediated lysis and induces apoptosis. Cancer Res. 1999:59:1287-94.

29. Ibraheem K, Yhmed AMA, Qayyum T, Bryan NP, Georgopoulos NT. CD40 induces renal cell carcinoma-specific differential regulation of TRAF proteins, ASK1 activation and JNK/p38-mediated, ROS-dependent mitochondrial apoptosis. Cell Death Discov. 2019;5(1):148. https://doi.org/10.1038/s41420019-0229-8.

30. Georgopoulos NT, Steele LP, Thomson MJ, Selby PJ, Southgate J, Trejdosiewicz LK. A novel mechanism of CD40-induced apoptosis of carcinoma cells involving TRAF3 and JNKJAP-1 activation. Cell Death Differ. 2006;13(10):1789-801. https://doi.org/10.1038/sj.cdd.4401859.

31. Eliopoulos AG, Davies C, Knox PG, Gallagher NJ, Afford SC, Adams DH, et al. CD40 induces apoptosis in carcinoma cells through activation of cytotoxic ligands of the tumor necrosis factor superfamily. Mol Cell Biol. 2000;20(15): 5503-15. https://doi.org/10.1128/MCB.20.15.5503-5515.2000.

32. Qiu X, Klausen C, Cheng JC, Leung PC. CD40 ligand induces RIP1dependent, necroptosis-like cell death in low-grade serous but not serous borderline ovarian tumor cells. Cell Death Dis. 2015;6(8):e1864. https://doi. org/10.1038/cddis.2015.229.

33. Hill SC, Youde SJ, Man S, Teale GR, Baxendale AJ, Hislop A, et al. Activation of CD40 in cervical carcinoma cells facilitates CTL responses and augments chemotherapy-induced apoptosis. J Immunol. 2005;174(1):41-50. https://doi. org/10.4049/jimmunol.174.1.41.

34. Knox PG, Davies CC, loannou M, Eliopoulos AG. The death domain kinase RIP1 links the immunoregulatory CD40 receptor to apoptotic signaling in carcinomas. J Cell Biol. 2011;192(3):391-9. https://doi.org/10.1083/jcb.201003087.

35. Pirozzi G, Lombari V, Zanzi D, lonna F, Lombardi ML, Errico S, et al. CD40 expressed on human melanoma cells mediates T cell co-stimulation and tumor cell growth. Int Immunol. 2000;12(6):787-95. https://doi.org/10.1093/ intimm/12.6.787.

36. Forbes SA, Bhamra G, Bamford S, Dawson E, Kok C, Clements J, Menzies A, Teague JW, Futreal PA, Stratton MR: The catalogue of somatic mutations in cancer (COSMIC). Curr Protoc Hum Genet 2008, Chapter 10:Unit 10-11.

37. Farooqi AA, Li KT, Fayyaz S, Chang YT, Ismail M, Liaw CC, et al. Anticancer drugs for the modulation of endoplasmic reticulum stress and oxidative stress. Tumour Biol. 2015;36(8):5743-52. https://doi.org/10.1007/s13277-015-3797-0.

38. Ritt DA, Abreu-Blanco MT, Bindu L, Durrant DE, Zhou M, Specht SI, et al. Inhibition of Ras/Raf/MEK/ERK pathway signaling by a stress-induced phospho-regulatory circuit. Mol Cell. 2016;64(5):875-87. https://doi.org/10.1 016/j.molcel.2016.10.029.

39. Aaes TL, Vandenabeele P. The intrinsic immunogenic properties of cancer cell lines, immunogenic cell death, and how these influence host antitumor immune responses. Cell Death Differ. 2021;28(3):843-60. https://doi.org/10.1 038/s41418-020-00658-y.

40. Waithman J, Gebhardt T, Bedoui S. Skin tumor immunity: site does matter for antigen presentation by DCs. Eur J Immunol. 2016;46(3):543-6. https:// doi.org/10.1002/eji.201646293.

41. Choi J, Beaino W, Fecek RJ, Fabian KPL, Laymon CM, Kurland BF, et al. Combined VLA-4-targeted radionuclide therapy and immunotherapy in a mouse model of melanoma. J Nucl Med. 2018;59(12):1843-9. https://doi. org/10.2967/jnumed.118.209510.

42. Meng Y, Harlin H, O'Keefe JP, Gajewski TF. Induction of cytotoxic granules in human memory CD8+ T cell subsets requires cell cycle progression. J Immunol. 2006;177(3):1981-7. https://doi.org/10.4049/jimmunol.177.3.1981.

43. LaFleur MW, Muroyama Y, Drake CG, Sharpe AH. Inhibitors of the PD-1 pathway in tumor therapy. J Immunol. 2018;200(2):375-83. https://doi.org/1 0.4049/jimmunol.1701044.

44. Khan AR, Hams E, Floudas A, Sparwasser T, Weaver CT, Fallon PG. PD-L1hi B cells are critical regulators of humoral immunity. Nat Commun. 2015;6(1): 5997. https://doi.org/10.1038/ncomms6997.

45. Guan $H$, Lan $Y$, Wan $Y$, Wang $Q$, Wang $C$, Xu L, et al. PD-L1 mediated the differentiation of tumor-infiltrating CD19(+) B lymphocytes and T cells in invasive breast cancer. Oncoimmunology. 2016;5(2):e1075112. https://doi. org/10.1080/2162402X.2015.1075112.

46. Yang J, Yan C, Vilgelm AE, Chen SC, Ayers GD, Johnson CA, et al. Targeted deletion of CXCR2 in myeloid cells alters the tumor immune environment to improve antitumor immunity. Cancer Immunol Res. 2021;9(2):200-13. https://doi.org/10.1158/2326-6066.CIR-20-0312.

47. Cunningham AF, Flores-Langarica A, Bobat S, Dominguez Medina CC, Cook $\mathrm{CN}$, Ross EA, et al. B1b cells recognize protective antigens after natural infection and vaccination. Front Immunol. 2014;5:535. 
48. Yang J, Hawkins OE, Barham W, Gilchuk P, Boothby M, Ayers GD, et al. Myeloid IKKbeta promotes antitumor immunity by modulating CCL11 and the innate immune response. Cancer Res. 2014;74(24):7274-84. https://doi. org/10.1158/0008-5472.CAN-14-1091.

49. Yang $Y$, Wilson JM. CD40 ligand-dependent T cell activation: requirement of B7-CD28 signaling through CD40. Science. 1996;273(5283):1862-4. https:// doi.org/10.1126/science.273.5283.1862.

50. Bugeon L, Dallman MJ. Costimulation of T cells. Am J Respir Crit Care Med 2000;162(supplement_3):S164-8. https://doi.org/10.1164/ajrccm.162. supplement_3.15tac5.

51. Shattuck-Brandt RL, Chen SC, Murray E, Johnson CA, Crandall H, O'Neal JF, et al. Metastatic melanoma patient-derived xenografts respond to $\mathrm{mdm} 2$ inhibition as a single agent or in combination with BRAF/MEK inhibition. Clin Cancer Res. 2020;26(14):3803-18. https://doi.org/10.1158/1078-0432. CCR-19-1895.

52. Chen PL, Roh W, Reuben A, Cooper ZA, Spencer CN, Prieto PA, et al. Analysis of immune signatures in longitudinal tumor samples yields insight into biomarkers of response and mechanisms of resistance to immune checkpoint blockade. Cancer Discov. 2016;6(8):827-37. https://doi.org/10.11 58/2159-8290.CD-15-1545.

53. Riaz N, Havel JJ, Makarov V, Desrichard A, Urba WJ, Sims JS, et al. Tumor and microenvironment evolution during immunotherapy with nivolumab. Cell. 2017;171(4):934-49 e916. https://doi.org/10.1016/j.cell.2017.09.028.

54. Jerby-Arnon L, Shah P, Cuoco MS, Rodman C, Su MJ, Melms JC, et al. A cancer cell program promotes $T$ cell exclusion and resistance to checkpoint blockade. Cell. 2018;175(4):984-97 e924. https://doi.org/10.1016/j.cell.2018.09.006.

55. Baker SJ, Cosenza SC, Athuluri-Divakar S, Reddy MVR, Carpio RV, Jain R, Aggarwal AK, Reddy EP. Mechanism of action of rigosertib does not involve tubulin binding. BioRxiv 2019

56. Nair AB, Jacob S. A simple practice guide for dose conversion between animals and human. J Basic Clin Pharm. 2016;7(2):27-31. https://doi.org/1 0.4103/0976-0105.177703.

57. Fogarty CE, Bergmann A. Killers creating new life: caspases drive apoptosisinduced proliferation in tissue repair and disease. Cell Death Differ. 2017; 24(8):1390-400. https://doi.org/10.1038/cdd.2017.47.

58. Diwanji N, Bergmann A. An unexpected friend - ROS in apoptosis-induced compensatory proliferation: implications for regeneration and cancer. Semin Cell Dev Biol. 2018:80:74-82. https://doi.org/10.1016/j.semcdb.2017.07.004.

59. Dunnill CJ, Ibraheem K, Mohamed A, Southgate J, Georgopoulos NT. A redox state-dictated signalling pathway deciphers the malignant cell specificity of CD40-mediated apoptosis. Oncogene. 2017;36(18):2515-28. https://doi.org/10.1038/onc.2016.401.

60. Beatty GL, Li Y, Long KB. Cancer immunotherapy: activating innate and adaptive immunity through CD40 agonists. Expert Rev Anticancer Ther. 2017;17(2):175-86. https://doi.org/10.1080/14737140.2017.1270208.

61. Long KB, Gladney WL, Tooker GM, Graham K, Fraietta JA, Beatty GL. IFNgamma and CCL2 cooperate to redirect tumor-infiltrating monocytes to degrade fibrosis and enhance chemotherapy efficacy in pancreatic carcinoma. Cancer Discov. 2016;6(4):400-13. https://doi.org/10.1158/2159-82 90.CD-15-1032.

62. Singh M, Vianden C, Cantwell MJ, Dai Z, Xiao Z, Sharma M, et al. Intratumoral CD40 activation and checkpoint blockade induces T cellmediated eradication of melanoma in the brain. Nat Commun. 2017:8(1): 1447. https://doi.org/10.1038/s41467-017-01572-7.

63. Dempke WCM, Fenchel K, Uciechowski P, Dale SP. Second- and thirdgeneration drugs for immuno-oncology treatment-the more the better? Eur J Cancer. 2017;74:55-72. https://doi.org/10.1016/j.ejca.2017.01.001.

64. Irenaeus SMM, Nielsen D, Ellmark P, Yachnin J, Deronic A, Nilsson A, et al. First-in-human study with intratumoral administration of a CD40 agonistic antibody, ADC-1013, in advanced solid malignancies. Int J Cancer. 2019; 145(5):1189-99. https://doi.org/10.1002/ijc.32141.

65. Knorr DA, Dahan R, Ravetch JV. Toxicity of an fc-engineered anti-CD40 antibody is abrogated by intratumoral injection and results in durable antitumor immunity. Proc Natl Acad Sci U S A. 2018;115(43):11048-53. https://doi.org/10.1073/pnas.1810566115.

66. Litchfield K, Reading JL, Puttick C, Thakkar K, Abbosh C, Bentham R, et al. Meta-analysis of tumor- and $T$ cell-intrinsic mechanisms of sensitization to checkpoint inhibition. Cell. 2021;184(3):596-614 e514. https://doi.org/10.101 6/j.cell.2021.01.002.
67. Jost M, Chen Y, Gilbert LA, Horlbeck MA, Krenning L, Menchon G, et al. Combined CRISPRi/a-based chemical genetic screens reveal that rigosertib is a microtubule-destabilizing agent. Mol Cell. 2017;68(1):210-23 e216. https://doi.org/10.1016/j.molcel.2017.09.012.

68. Jost M, Chen Y, Gilbert LA, Horlbeck MA, Krenning L, Menchon G, et al. Pharmaceutical-grade rigosertib is a microtubule-destabilizing agent. Mol Cell. 2020;79(1):191-8 e193. https://doi.org/10.1016/j.molcel.2020.06.008.

69. Vilgelm AE, Pawlikowski JS, Liu Y, Hawkins OE, Davis TA, Smith J, et al. Mdm2 and aurora kinase a inhibitors synergize to block melanoma growth by driving apoptosis and immune clearance of tumor cells. Cancer Res. 2015;75(1):181-93. https://doi.org/10.1158/0008-5472.CAN-14-2405.

70. Yang J, Kumar A, Vilgelm AE, Chen SC, Ayers GD, Novitskiy SV, et al. Loss of CXCR4 in myeloid cells enhances antitumor immunity and reduces melanoma growth through NK cell and FASL mechanisms. Cancer Immunol Res. 2018;6(10):1186-98. https://doi.org/10.1158/2326-6066.CIR-18-0045.

71. Olivo Pimentel V, Yaromina A, Marcus D, Dubois LJ, Lambin P. A novel coculture assay to assess anti-tumor CD8(+) T cell cytotoxicity via luminescence and multicolor flow cytometry. J Immunol Methods. 2020;487: 112899. https://doi.org/10.1016/j.jim.2020.112899.

72. Love Ml, Huber W, Anders S. Moderated estimation of fold change and dispersion for RNA-seq data with DESeq2. Genome Biol. 2014;15(12):550. https://doi.org/10.1186/s13059-014-0550-8.

73. LBenjamini $Y$, Hochberg Y. Controlling the false discovery rate: a practical and powerful approach to multiple testing. J R Statist Soc B. 1995;57(1):289-300.

\section{Publisher's Note}

Springer Nature remains neutral with regard to jurisdictional claims in published maps and institutional affiliations.

\section{Ready to submit your research? Choose BMC and benefit from:}

- fast, convenient online submission

- thorough peer review by experienced researchers in your field

- rapid publication on acceptance

- support for research data, including large and complex data types

- gold Open Access which fosters wider collaboration and increased citations

- maximum visibility for your research: over $100 \mathrm{M}$ website views per year

At BMC, research is always in progress.

Learn more biomedcentral.com/submissions 